\title{
Coupled Oscillators Model of Hyperexcitable Neuroglial Networks
}

by

Firas H. Farah

A thesis submitted in conformity with the requirements

for the degree of Master of Applied Science

Edward S. Rogers Sr. Department of Electrical and Computer Engineering University of Toronto

(C) Copyright by Firas H. Farah 2017 


\title{
Coupled Oscillators Model of Hyperexcitable Neuroglial Networks
}

\author{
Firas H. Farah \\ Master of Applied Science \\ Edward S. Rogers Sr. Department of Electrical and Computer Engineering \\ University of Toronto
}

2017

\begin{abstract}
Presented in this thesis is an oscillator-based neuroglial model capable of generating Spontaneous Electrical Discharges (SEDs) in hyperexcitable conditions. The network is composed of 16 coupled Cognitive Rhythm Generators (CRGs), which are mathematical constructs that represent one of the following populations of excitable cells: an excitatory pyramidal cell population, an inhibitory interneuron population, an astrocyte population, or a microglial population. Various mechanisms leading to hyperexcitability were investigated, and results suggest an important role for astrocytes and microglia in the generation of SEDs of various durations. Analysis of the resultant SEDs revealed two underlying distributions with differing properties. Particularly, short and long SEDs are associated with deterministic and random processes, respectively. Furthermore, there was evidence of theta-HFO phase-amplitude cross-frequency coupling (CFC) in the short SEDs, and delta-HFO CFC in the long SEDs, as was previously reported in a mouse model of Seizure-Like Events (SLEs) and in human patients with epilepsy.
\end{abstract}




\section{Acknowledgments}

I'd like to thank all of the lab members for their ideas and support, and for making the lab an enjoyable place. Particularly, thanks to Daniel, John, Mark, Sinisa, Trevor, Uilki, Vanessa, and Vasily. I'd also like to thank Dr. Zalay. The quality of your work has been truly inspiring.

I'd like to thank my supervisor Dr. Bardakjian for the constant support and guidance. Most of all, thank you for teaching me to not indulge my anxieties and to be comfortable living with a degree of uncertainty. Your supervision has made this experience a valuable one, in ways far beyond coupled oscillators.

I'd like to thank my friends, especially Yuma, for being a constant source of calm and positivity. I am very thankful for my family for all of their love and support. Above all, I am thankful for my mother, who has experienced my highs and lows more intensely than I have. I am truly lucky to have lived to witness and experience such a bond. No words or actions could ever express my gratitude. 


\section{Table of Contents}

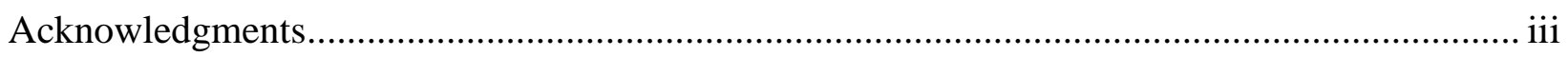

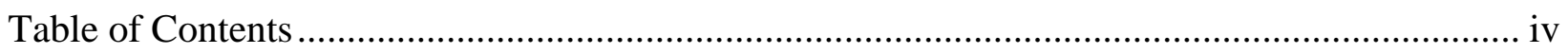

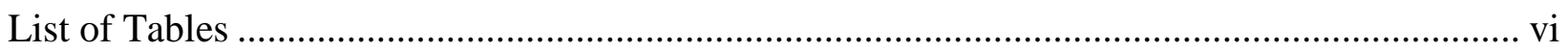

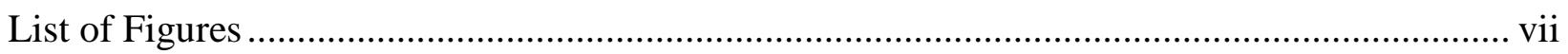

List of Abbreviations ........................................................................................................... vii

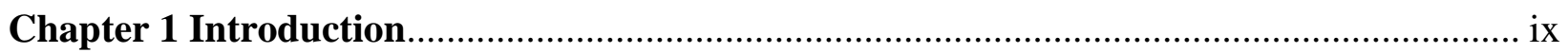

1.1 Epilepsy

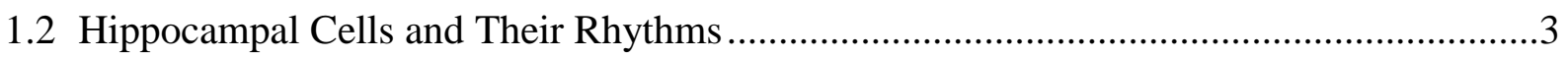

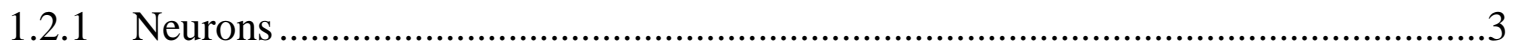

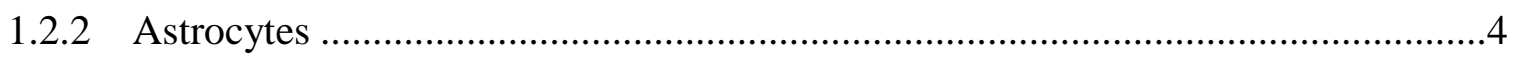

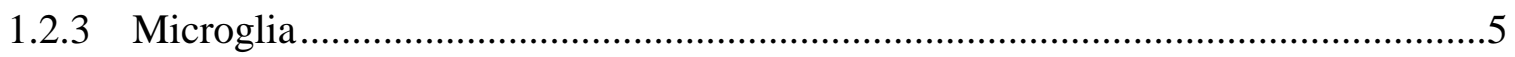

1.2.4 Cross-Frequency Coupling (CFC) and Neural Coding............................................

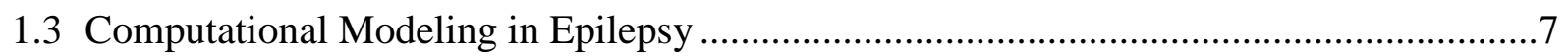

1.4 Objective and Hypotheses........................................................................................... 10

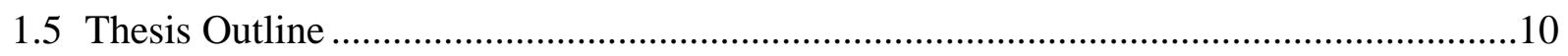

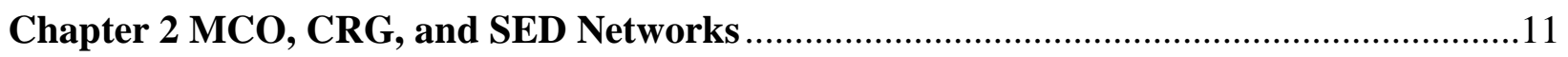

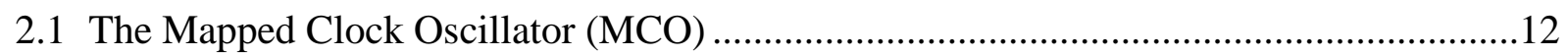

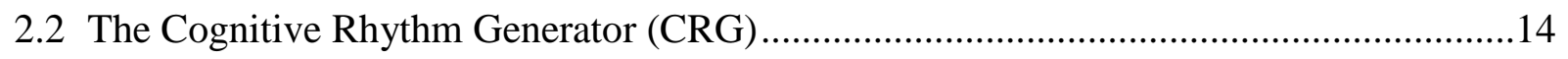

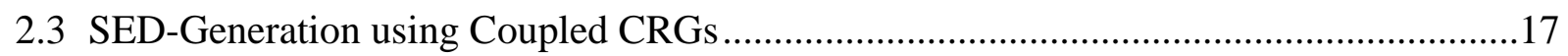

2.3.1 The Original SED Network ...........................................................................17

2.3.2 The HFO SED Network..............................................................................18

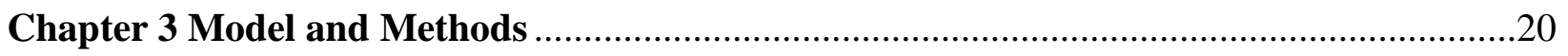

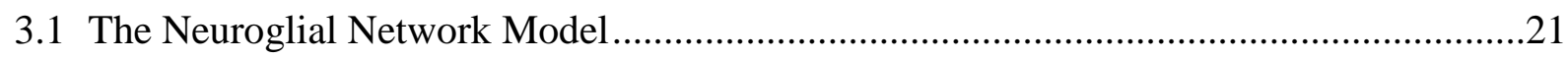

3.1.1 Mathematics of the Network Model ................................................................21 
3.1.2 Connectivity and Features of the Network Model ..........................................24

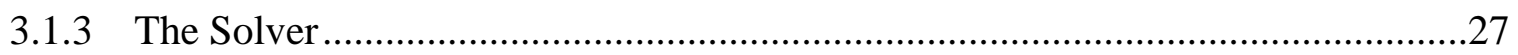

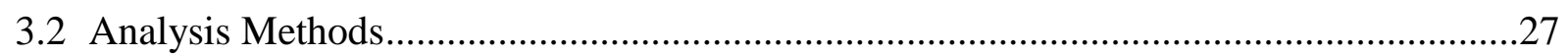

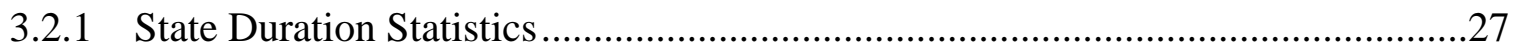

3.2.2 Quantifying the Excitability Level of the System........................................28

3.2.3 LFO-HFO Phase-Amplitude Coupling ......................................................29

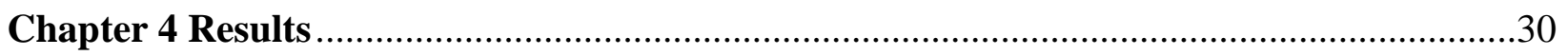

4.1 The Network Model Generates Recurrent SEDs ......................................................... 31

4.2 Different Processes Govern System Transitions in Short and Long Duration SEDs ........32

4.3 Glia-Neuron Interactions Play an Important Role in Controlling Hyperexcitability .........34

4.4 Several Mechanisms Can Lead to Hyperexcitability .................................................37

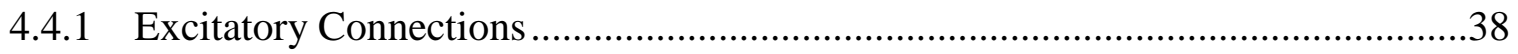

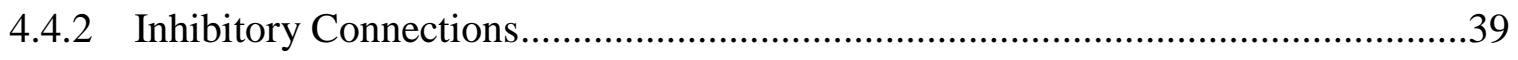

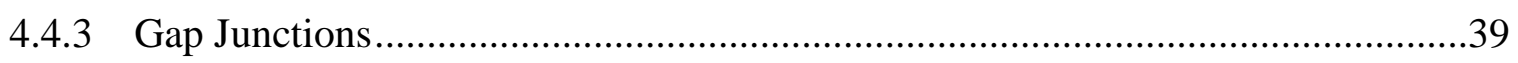

4.5 1SEDs Exhibit Delta-HFO and sSEDs Exhibit Theta-HFO Phase-Amplitude Coupling ..42

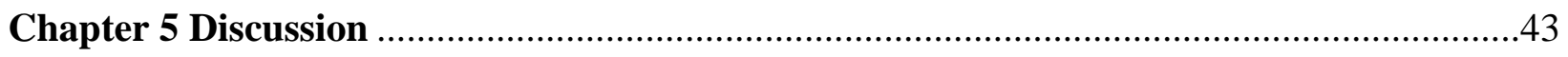

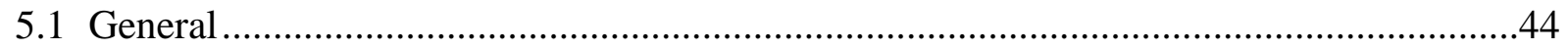

5.2 Glial Cells Play an Important Role in Hyperexcitability ...........................................44

5.3 Comparison of Short and Long SEDs...........................................................

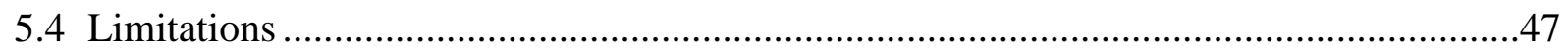

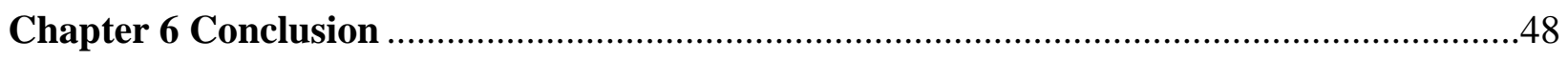

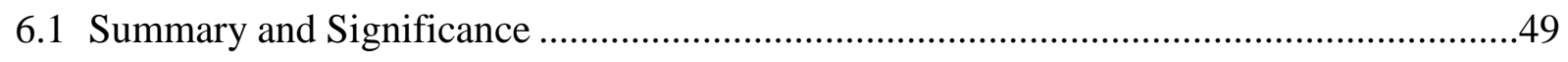

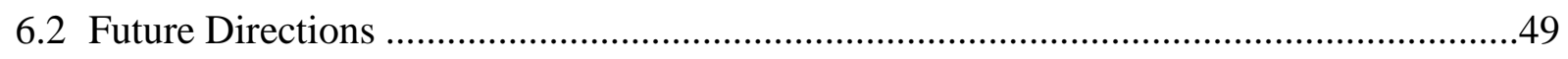

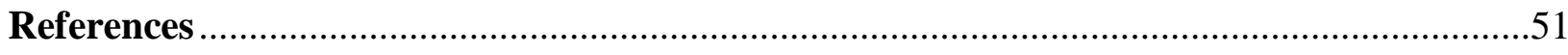

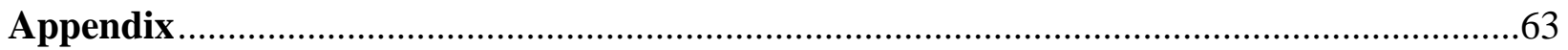




\section{List of Tables}

Table 3.1 Neuroglial network model coupling coefficients .................................26

Table 3.2 Neuroglial network model parameters........................................26

Table 4.1 Association of connection type with percent change in excitability..................38 


\section{List of Figures}

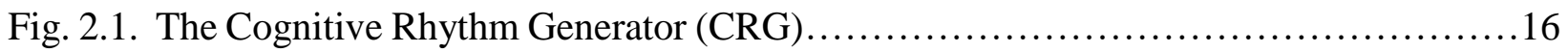

Fig. 2.2. Behavior of the clock and labile clock ring devices............................ 17

Fig. 2.3. Spontaneous recurrent SEDs in a network of 4 coupled CRGs.....................18

Fig. 2.4. Model schematic of the HFO SED model.....................................19

Fig. 2.5. Spontaneous recurrent SEDs as observed from the model outputs.................20

Fig. 2.6. Comparison of the HFO SED model outputs with recordings from the low- $\mathrm{Mg}^{2+} /$ high-

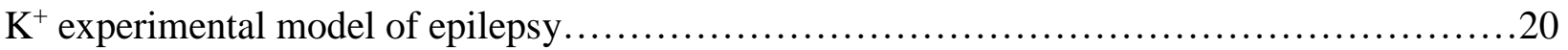

Fig. 3.1. The CRG neuroglial network model.......................................24

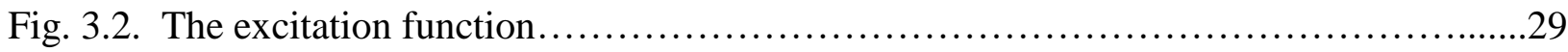

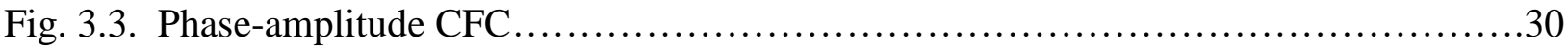

Fig. 4.1. Outputs of the network model under conditions of hyperexcitability.................32

Fig. 4.2. Results of a Gaussian Mixture Model applied to SED duration data..................33

Fig. 4.3. Histograms and fitted gamma functions for SED and inter-SED duration data........34

Fig. 4.4. Glial effects on network response as demonstrated from the LFP..................36

Fig. 4.5. Microglial pruning effect at various levels of system excitability as seen from the

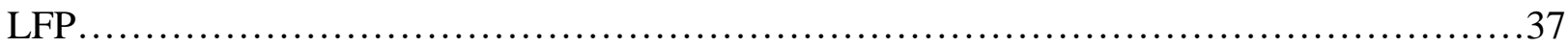

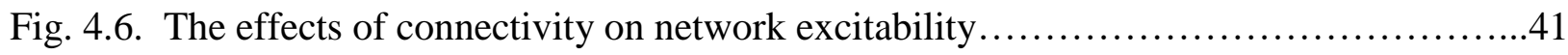

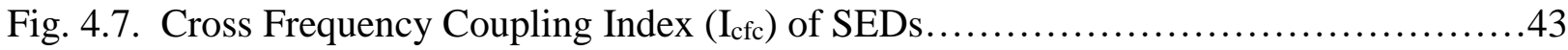

Fig. A.1. The sigmoidal pruning mechanism........................................65 


\section{List of Abbreviations}

$\mathrm{ACh}$

AED

AMPA

AQP

ATP

CA

CFC

CNS

CRG

EEG

GABA

GMM

HFO

$\mathrm{I}_{\mathrm{cfc}}$

ILAE

Kir

LFO

LFP

LTD
Acetylcholine

Anti-Epileptic Drug

$\alpha$-amino-3-hydroxy-5-methyl-4-isoxazolepropionic acid

Aquaporin

Adenosine Triphosphate

Cornu Ammonis

Cross-Frequency Coupling

Central Nervous System

Cognitive Rhythm Generator

Electroencephalogram

Gamma-Aminobutyric acid

Gamma Mixture Model

High Frequency Oscillations

Index of Cross-Frequency Coupling

International League Against Epilepsy

Inward Rectifying Potassium Channel

Low Frequency Oscillations

Local Field Potential

Long Term Depression 
LTP

$\mathrm{MCO}$

NFM

NMDA

NMM

PAC

PDM

SED / sSED / 1SED

SEM

SLE

TLE
Long Term Potentiation

Mapped Clock Oscillator

Neural Field Model

N-methyl-D-aspartate

Neural Mass Model

Phase Amplitude Coupling

Principal Dynamic Modes

Spontaneous Electrical Discharges / short SED / long SED

Standard Error of the Mean

Seizure-Like Event

Temporal Lobe Epilepsy 


\section{Chapter 1}

\section{Introduction}

\subsection{Epilepsy}

Epilepsy is a dynamical disease with a wide spectrum of causes and symptoms, and is characterized by recurrent seizures [1] [2]. A seizure is defined by the International League against Epilepsy (ILAE) as a "transient occurrence of signs and/or symptoms due to abnormal excessive or synchronous neuronal activity in the brain," and can come abruptly without warning [3]. Epilepsy is highly prevalent affecting a large number of individuals, with sources stating statistics, such as 50 million individuals affected worldwide and $1 \%$ of the world's population [4] [5]. It is the $4^{\text {th }}$ most common neurological disorder in the United States, and 1 in every 26 people will develop epilepsy at some point in their lives [6].

Seizures are most generally categorized into two classes based on the spatial extent of the activity in the brain [7]. The first class, known as partial or focal seizures, affects only a part of one hemisphere, and there is a "focus" where the seizure begins. These can occur with retained awareness (formerly known as simple partial seizures), where the patient's consciousness is unimpaired, or with a loss of awareness (formerly known as complex seizures). This latter kind often originates in the temporal or frontal lobe of the brain and causes the patient to become unaware of the surrounding environment and to perform meaningless physical actions [8].

The second class of seizures is referred to as "generalized," where the activity is more widespread and affects both hemispheres of the brain [9]. There are several seizure types within this class, differing in clinical behavior and in the electroencephalogram (EEG) [10]. One type, the absence seizure (formerly known as petit mal), results in unconsciousness, without convulsions. These appear in the EEG as discharges lasting 1 to 20 seconds in durations, with spike-wave complexes occurring at a frequency of 3-4 Hz [10]. Another type, the tonic-clonic convulsive seizure (formerly known as grand mal), results in a loss of consciousness, and consist of two phases. In the tonic phase, muscles stiffen often causing the patient to fall, and it usually appears in the EEG as a rhythmic 10-12 Hz (alpha) rhythm [10]. In the clonic phase, the extremities jerk and twitch, 
and the EEG is characterized by polyspike-and-wave complexes [10]. Abnormally prolonged seizures can lead to status epilepticus, and can have long-term consequences, such as neuronal death, neuronal injury, and alteration of neuronal networks [11].

There are many different causes of epilepsy, and these are largely dependent on the individual's genetic susceptibilities [12]. The causes are different depending on the time of onset. An onset in childhood is predominantly genetically determined. Variants of the SCN1A gene, for example, can lead to a treatment-resistant epilepsy, known as Dravet Syndrome [13]. In adults, the causes are usually related to a structural change, such as hippocampal sclerosis, cortical dysplasia, and arteriovenous malformations [12]. Structural changes could be brought about in different ways, such as head injury and infectious causes of encephalitis [14] [15].

The consequences of epilepsy cause patients to experience a lower quality-of-life [16]. A health study found that "patients had significantly lower scores in ...: general health perceptions, mental health, vitality, role limitations owing to physical and emotional problems, and social functioning" [17]. Patients often experience driving restrictions, trouble seeking and holding jobs, going to school, and obtaining insurance coverage [18]. The psychosocial problems can be more handicapping than the seizures themselves, and can lead to social isolation due to stigmatization.

The first option for the treatment of epilepsy is usually anti-epileptic drugs (AEDs). These pharmacological solutions often have side-effects, and 30\% of all patients fail to respond [19] . Surgical intervention may be considered in these refractory cases. Candidates for surgery undergo a diagnostic evaluation involving EEG analysis and structural and functional imaging to find the epileptogenic tissue to be resected [20]. In addition to removing tissue containing the epileptogenic zone, surgery may be used to cut tissue to prevent the spread of seizure activity, as in the case of subpial transection and corpus callosatomy [21]. Surgical intervention, however, is not always an adequate solution. Not all patients are candidates for surgery, as with those with multifocal seizure origin [22]. Furthermore, surgeries are not always successful, particularly if the patient has had epilepsy for a long period of time [22]. Surgeries can be accompanied by surgical and neurological complications, and in some cases, if the surgery is not successful in eliminating seizures, reoperation may be necessary [23] [24] [25]. Electrical stimulation is yet another option for drug-resistant epilepsy. Vagal Nerve Stimulation (VNS), Deep Brain Stimulation (DBS), and Transcranial Magnetic Stimulation (TMS) have all recently been tested in clinical trials, to varying 
levels of success [26] [27] [28]. They are however relatively new, and have yet to reach widespread clinical use [29] [30].

\subsection{Hippocampal Cells and Their Rhythms}

The hippocampus is an important structure for learning and memory, and it has contributed immensely to our understanding of elemental brain circuits [31] [32]. It is most often divided into four subfields: CA1, CA2, CA3, and CA4. The hippocampus can generate Low Frequency Oscillations (LFOs) below $30 \mathrm{~Hz}$ and High Frequency Oscillations (HFOs) faster than $30 \mathrm{~Hz}$ [33] [34]. It is heavily studied in the context of epilepsy, and is often considered to be the generator of Temporal Lobe Epilepsy (TLE) [35]. The sections below contain brief descriptions of relevant cellular populations, their rhythms, and their potential roles in hyperexcitability and epilepsy.

\subsubsection{Neurons}

The hippocampus consists of a wide variety of neuronal populations, with pyramidal cells and interneurons being two important classes. Pyramidal cells are largely excitatory in nature and can fire action potentials over a wide range of frequencies [36]. They are known to exhibit spontaneous and evoked intrinsic burst firing [37]. In the CA3, pyramidal neurons excite one another and excite interneurons [38]. In contrast to pyramidal cells, interneurons are largely inhibitory in nature, predominantly using GABA as their neurotransmitter [39]. There are a wide variety of different interneuron types, such as fast-spiking basket cells and O-LM interneurons [32]. In the hippocampus, interneurons can perform feedback and feedforward inhibition onto the pyramidal populations, as well as mutual inhibition among one another [32]. They can oscillate at a wide range of frequencies, some faster than $300 \mathrm{~Hz}$ [40]. Furthermore, certain classes of interneurons are known to be involved in the induction and maintenance of hippocampal network oscillations in the theta, gamma, and ultrafast rhythms (> $200 \mathrm{~Hz})$ [40].

Neurons are the most extensively studied cellular populations in epilepsy, and the interaction between pyramidal cells and interneurons has formed the basis of a large number of studies [41] [42] [43]. A classical and prevalent hypothesis is that epilepsy is caused in part by a pathological disruption in the balance between neuronal excitation and inhibition [44] [45]. Krook-Magnuson et al., for example, have demonstrated that the optogenetic inhibition of excitatory principal cells 
or the excitation of GABAergic hippocampal cells stops seizures rapidly [46]. Furthermore, antagonists of glutamate NMDA receptors are known to be anti-convulsant, whereas antagonists of GABA produce seizures [47] [48]. This principal underlies the development of many AEDs, such as perampanel (AMPA receptor antagonist) and vigabatrin (inhibitor of GABA breakdown) [49] [48].

\subsubsection{Astrocytes}

Astrocytes are star-shaped cells that have been traditionally thought of as "brain glue" providing a scaffold for neuronal distribution and playing a supporting role for neurons [50]. Intracellular recordings from astrocytes reveal the presence of slow oscillations, particularly in the delta frequency range [51] [52]. Astrocytes are important for supplying nutrients, neuronal metabolism, repair, synaptogenesis, and cerebral microcirculation [53]. More recently, however, insights have been gained into a broader and more complex role for astrocytes, and numerous potential mechanisms leading to pathologies, such as epilepsy, have been identified.

One of the first recognized contributions of astrocytes to hyperexcitability is the impaired uptake of $\mathrm{K}^{+}$ions [54]. During neuronal discharges, there is an increase in the extracellular $\mathrm{K}^{+}$ concentration, which if left to accumulate, can cause excessive neuronal discharges and can lead to seizures. Normally, astrocytes can remove large amounts of the extracellular $\mathrm{K}^{+}$in a number of ways, the most notable of which is the inward rectifying potassium (Kir) channel. Dysfunctional Kir channels have been linked to increased hyperexcitability in experimental animal models and in human patients. For example, Djukic et al. found that Kir4.1 knockout mice display severe seizures and die prematurely [55]. Similarly, various studies of epileptic human patients have found a loss or change in Kir4.1 channels and potassium conductance [56] [57]. Dysfunctional gap junctions have also been implicated in high extracellular $\mathrm{K}^{+}$levels, due to the inability of the astrocytes to redistribute the potassium ions throughout the glial syncytium [58].

Working in conjunction with potassium channels, aquaporins are important for maintaining water balance in astrocytes and the extracellular space [59]. Aquaporin 4 (AQP4) is one of 11 family integral proteins that mediate the transport of water across cellular membranes. They are colocalized with Kir4.1 channels near the perivascular space and allow water to move into the capillaries. Under pathological conditions, the astrocytes can swell thus reducing the size of the extracellular space. This is known to favor hyperexcitability by allowing for stronger ephaptic 
connections between neurons, as well as altered ion and neurotransmitter concentrations and diffusion rates [60]. Furthermore, the removal of the perivascular AQP4 has been shown to compromise the $\mathrm{K}^{+}$buffering capacity of astrocytes, and electron microscopy revealed a reduced density of the perivascular AQP4 in the human epileptogenic hippocampus [61].

In addition to $\mathrm{K}^{+}$ions, astrocytes are responsible for the uptake and metabolism of neurotransmitters, such as glutamate [62]. Glutamate is an excitatory neurotransmitter that can lead to excessive neuronal activity and the occurrence of seizures. Eid et al. found that a key enzyme required for the metabolism of glutamate is reduced in epileptic patients and hypothesized that this may lead to higher extracellular concentrations [63].

Astrocytes have even been shown to release glutamate in response to elevations in internal $\mathrm{Ca}^{2+}$ levels. They are able to modulate the amplitude of their $\mathrm{Ca}^{2+}$ signal in response to different synapses that use different transmitters, such as glutamate and acetylcholine (ACh) [64]. Then, using one of various mechanisms, such as vesicle formation or reversal of transporters, they can release substances like glutamate and D-serine. This release has not only been shown to strengthen synapses, but also to mediate neuronal synchronizations that predisposes the tissue to seizures [65].

\subsubsection{Microglia}

Microglia are known as the resident immune cells of the nervous system [66]. Their processes are highly motile and constantly extend and retract allowing them to actively gauge the health of surrounding cells [67]. They constantly survey the brain for damage and are responsible for engulfing dead cells and debris [66]. Furthermore, they are important in processes required for learning, memory, and cognitive function [68]. During development, they are known to play a role in regulating neurogenesis, in promoting neural precursor cell development, and in enhancing neuronal survival [69] [70]. In the mature CNS, microglia can regulate long term potentiation (LTP), synaptic scaling, and basal glutamergic and GABAergic transmission [71] [72] [73]. For

example, microglia can release ATP that binds on astrocytes and causes an increase in excitatory transmission via a metabotropic glutamate receptor-dependent mechanism [73].

An important role for microglia, particularly during development, is their involvement in synaptic remodeling. In response to neuronal activity, microglia can "prune" or "strip" synapses through a phagocytic mechanism [74]. Microglia physically ensheathe neurons and insert processes into the 
synaptic cleft to separate pre and post synaptic elements [75]. The contact of the microglia with neuronal elements is activity-dependent and the synaptic stripping is thought to be due to reduced electrical activity [76]. Wake et al., however, found that by performing binocular enucleation and thus reducing neuronal activity in layer II/III of the visual cortex, microglial processes retracted and there was a reduced contact frequency with synapses [75]. Microglia are known to respond to neuronal signals, and have receptors for ACh, GABA, ATP, and glutamate [77] [71]. Fontainhas et al. reported that excitatory neurotransmission increases microglial motility, while inhibitory neurotransmission decreases motility, and concluded that these are indirectly mediated by ATP signaling [78].

In epilepsy, microglia undergo reactive gliosis, a component of neuroinflammation involving structural and metabolic changes. Following seizures, changes in the morphological characteristics of microglia, such as increased numbers, increased cell body size, fewer processes, and decreased process branching have been reported [69]. In addition, increased expression of certain proteins, such as fractalkine receptors, have been observed in epileptic patients [79]. (Fractalkine receptors are selectively expressed on microglia and the fractalkine chemokine is expressed by neurons, facilitating a privileged interaction between the two populations [80]).

Whether the role of microglia is harmful or helpful in epilepsy and seizures remains unclear. There is evidence that they are neuroprotective early in acute seizures [69]. Eyo et al. reported increased microglial contact with neuronal elements that when blocked resulted in a worsened seizure phenotype [81]. Furthermore, Derecki et al. found that blocking microglial phagocytosis promotes the development of Rett Syndrome, a condition that most often results in seizures [82]. Moreover, during CNS injury or in neurodegenerative diseases, microglia generally release cytokines to suppress neuroinflammation [83]. On the other hand, there is evidence for a harmful neurodegenerative role for microglia in epilepsy. Ali et al., for example, found that the inhibition of fractalkine signaling reduced neurodegeneration in the hippocampus [79]. In addition, microglia may contribute to seizure-induced aberrant neurogenesis [84].

\subsubsection{Cross-Frequency Coupling (CFC) and Neural Coding}

As mentioned above, the hippocampus is capable of generating intrinsic rhythms with a wide range of frequencies. The interaction of these rhythms forms a "code," that has been found to be crucial in various cognitive functions. There is evidence that the interaction of different rhythms can allow 
for the representation of higher order-brain states, is important in sensory and memory processes, and may underlie voluntary behavior [85] [86].

The interaction of the theta and gamma rhythms is often discussed in the hippocampus for its role in the readout of long-term memories. The theta-gamma neural code is usually described in the context of rodent place cells, where it was found that the spatial location of a rodent is encoded by the spatial pattern of neurons that fire in a gamma cycle, and the order of the locations is encoded in sequential gamma sub-cycles of the theta cycle [87]. These theta-gamma interactions have been observed in areas other than the hippocampus however, and are not restricted to the readout of long-term memories. Dual oscillations serve a general function of encoding multiple units of information in a way that preserves their serial order [86]. Of course, CFC is not limited to rodents, but has been observed in a variety of human functions, such as in working memory and in memory encoding and retrieval [88] [89].

One form of CFC is phase-amplitude coupling (PAC), which refers to the phenomenon where the amplitude of an HFO is modulated by the phase of an LFO [90] [91]. Phase-amplitude CFC, particularly between delta or theta rhythms and HFOs, has been found to exist in human seizures and in experimental animal SLEs [33] [92] [93] [94]. Furthermore, it has been practically used as a feature for the prediction and classification of seizure states and for the successful identification of the epileptogenic zone for resection [95] [96] [97] [33].

\subsection{Computational Modeling in Epilepsy}

As mentioned previously, available treatments for epilepsy are far from sufficient, as a) $30 \%$ of epileptic patients are pharmacoresistant, b) surgical interventions often require reoperation and patients may still not be seizure-free, and c) while electrical stimulation has shown some promise, it has yet to reach widespread clinical use. The development of effective treatments remains hampered by the complexity of the underlying systems and processes, and Wendling describes four reasons for this [98]:

1. The underlying mechanisms are usually non-linear and span multiple spatial scales from subcellular ionic channels to brain regions.

2. The recordings do not fully capture all underlying mechanisms. 
3. Our observations are incomplete in time and space. For example, recordings are done over a relatively short time window as compared to the overall progression of the disease.

4. Epileptic phenomena involve multiple time scales, from the duration of a spike (milliseconds) to the frequency of seizures (days or months).

Furthermore, the cause of seizures is multifactorial and can depend on multiple genetic factors and/or acquired insults [99]. As such, an integrative approach is necessary for understanding the underlying mechanisms, and computational modeling is a useful tool in understanding this complexity.

Computational models can be useful in many ways in the context of epilepsy [100]. They can elucidate potential mechanisms underlying the reproduced behavior of the model. By including features in the model that replicate certain epileptic phenomena, the source of the behavior can be better understood. Furthermore, they can be used to generate hypotheses and make predictions. These predictions can then be tested experimentally, and this will either validate the model or highlight the need for its improvement. Models can also be used to test hypotheses that may be difficult to assess experimentally. For example, it may be possible to simultaneously simulate and view the activity of hundreds of cellular populations, while it would be difficult to obtain intracellular recordings from that many cells experimentally. Similarly, they can provide an efficient platform upon which various treatments, from AEDs to stimulation, can be tested. Colic et al. and Zalay et al., for example, both tested different stimulation paradigms on a CRG-based SLE model (described in section 2.3.1) [101] [102].

Two general approaches to modeling exist: parametric and non-parametric modeling. Parametric modeling deals with the structural identification of a system, representing components in terms of a finite number of parameters in differential equations. Non-parametric modeling, on the other hand, deals with the functional identification of a system. The system is treated as a black-box that is "poked" to create an input-output map without concern for the internal structure. By probing the system with a stimulus and observing the response, it is possible to obtain Volterra and Weiner kernels, which provide a high-level description without specification of internal parameters [103] [104].

Parametric modeling of epileptiform activity can be done on multiple scales, ranging from subcellular levels to cortical regions [98]. At the microscopic scale, models explicitly represent the 
structure and function of cells and include parameters related to morphology, as well as passive and active electrical properties [100]. Neurons in these models are often based on adapted versions of the Hodgkin-Huxley equations, or on lower-order representations, such as the FitzhughNagumo relaxation oscillator [98] [105]. Van Drongelen et al., for example, explained mechanisms underlying seizure propagation in the neocortex by connecting various types of pyramidal cells and interneurons, represented by multi-compartmental models with biophysically realistic ion channels [106].

Mesoscopic parametric models deal with "lumped" descriptions of cellular assemblies or populations without concern for cellular-level mechanisms. While this abstraction serves to alleviate a potentially overwhelming computational task, they are still often based on neurophysiological concepts. Coupling constants, for example, can represent the average number of synaptic contacts between the cell types [100]. Suffczynksi et al. have developed one such Neural Mass Model (NMM), where the dynamics of non-convulsive epileptic phenomena were modeled using interconnected cortical and thalamic circuits [107].

The Cognitive Rhythm Generator (CRG), previously described and used by our research team, is a mathematical construct suitable for use in mesoscopic modeling. It is well-suited for modeling assemblies of excitable cells, and has been used to model various physiological phenomena, such as directional selectivity, phase preference, and phase precession [108]. Furthermore, a network of coupled CRGs has been used to model hippocampal neurons and to generate Spontaneous Electrical Discharges (SEDs) of similar characteristics to SLEs observed in a low- $\mathrm{Mg}^{2+} / \mathrm{high}^{+} \mathrm{K}^{+}$ experimental model of epilepsy. In brief, a CRG consists of 3 stages: i) Principal Dynamic Modes (PDMs) that perform decoding and transformation operations on incoming signals, ii) an oscillator ring device that generates instantaneous phase and amplitude values, and iii) a static non-linearity mapper that uses these values to generate an observable transmembrane voltage output. The development and mathematics of the CRG will be discussed in detail in Chapter 2.

NMMs typically deal with temporal dynamics, but not spatial evolution. Macroscopic Neural Field Models (NFMs) can account for the spatiotemporal evolution of various variables, and have been used to assist with the forward problem to better interpret the recordings obtained from epileptic human patients [100] [109]. 


\subsection{Objective and Hypotheses}

While there recently has been an interest in modeling glial cells in the context of epilepsy, few studies have taken an oscillator-based approach [110] [111] [112] [113]. The objective of this work is to develop a mesoscale neuroglial network model composed of coupled Cognitive Rhythm Generators (CRGs) that can give rise to Spontaneous Electrical Discharges (SEDs) with similar characteristics to those previously observed in SLEs and in human epileptic seizures.

The hypotheses of this thesis are:

1. A CRG-based neuroglial model is capable of generating short and long SEDs of various durations

2. Astrocytes and microglia both play an important role in modulating hyperexcitability and in the generation of SEDs

3. Different dynamics underlie the transitions into and out of short and long SEDs

4. The SEDs exhibit similar phase-amplitude CFC interactions to those observed in SLEs and in human epileptic seizures

\subsection{Thesis Outline}

The thesis is outlined as follows:

Chapter 1 - Introduction: This present chapter has provided some background information regarding epilepsy and hyperexcitability, and described the physiological relevance of different cellular populations and their interactions. It introduced computational modeling, and described the objective and hypotheses of this work.

Chapter 2 - MCO, CRG, and SED Networks: This chapter will describe the CRG and its predecessor, the Mapped Clock Oscillator (MCO). It will then describe two models where CRGs have been coupled to create SED-generating networks.

Chapter 3 - Model and Methods: This chapter will introduce the mathematics and features of the neuroglial network model that is the topic of this thesis. It will then briefly describe the techniques used to analyze the outputs of the model. 
Chapter 4 - Results: This chapter will highlight important results.

Chapter 5 - Discussion: This chapter will discuss the results of the model as it draws from relevant literature. It will then highlight the model's limitations.

Chapter 6 - Conclusion: This chapter will conclude the thesis by summarizing the results and discussing the significance of this work. The thesis will then close by providing various avenues for future investigation. 


\section{Chapter 2}

\section{MCO, CRG, and SED Networks}

The neuroglial model discussed in this thesis utilizes the Cognitive Rhythm Generator (CRG) as its base unit. This chapter will begin by briefly describing the Mapped Clock Oscillator (MCO), a predecessor to the CRG. It will then detail the development and mathematics of the CRG. Finally, it will describe how multiple CRGs have been coupled to create networks capable of generating SEDs, similar to the SLEs observed in experimental models of epilepsy.

\subsection{The Mapped Clock Oscillator (MCO)}

The MCO, developed by Bardakjian, is a Winfree-type oscillator that can be used to represent a population of excitable cells, such as gastric smooth muscle and neuronal populations [114] [115] [116] [117]. It consists of two stages: a ring that generates phase and amplitude values and a transformer that maps them into an observable transmembrane output.

Winfree-type oscillators are second-order oscillators, based on the concept of a "ring device," that focus on the rate of change of phase and amplitude. The unperturbed isolated intrinsic clock oscillator can be represented by:

$$
\begin{gathered}
\dot{\alpha}=\omega \alpha\left(1-\alpha^{2}\right) \\
\dot{\phi}=\omega
\end{gathered}
$$

where $\alpha$ is the intrinsic radial amplitude of the attracting cycle, $\phi$ is the intrinsic phase angle, and $\omega$ is the intrinsic angular frequency. Note that:

$$
\begin{aligned}
& \dot{\alpha}<0, \quad \text { if } \alpha>1 \\
& \dot{\alpha}=0, \quad \text { if } \alpha=0 \text { and } 1
\end{aligned}
$$




$$
\dot{\alpha}>0, \quad \text { if } 0<\alpha<1
$$

In all cases other than $\alpha=0$, solutions approach $\alpha=1$. As such, the unit circle is a limit cycle and the origin is an unstable focus.

$n$ oscillators can be coupled to simulate the interaction of $n$ populations of cells using $2 n$ differential equations. In doing so, the phase and amplitude of an oscillator will be stimulated as follows:

$$
\begin{gathered}
\dot{\alpha}=\omega \alpha\left(1+S_{\alpha}-\alpha^{2}\right) \\
\dot{\phi}=\omega\left(1+S_{\phi}\right)
\end{gathered}
$$

where $S_{\alpha}$ is a stimulus that affects mainly the amplitude of oscillation and $S_{\phi}$ mainly stimulates the phase. These oscillator dynamics form the first stage of the MCO.

In a coupled system, the stimuli will be given by:

$$
S_{k}=C_{k} R x
$$

where $k=\{\phi, \alpha\}, x$ is the input to the oscillator (which could be the output of another MCO), $C_{k}$ is the coupling coefficient between the input and the present oscillator taking a value between 0 and 1 , and $R$ is a refractory function that can be represented by a sigmoid or a highpass Butterworth function. This refractory function will prevent the ring from being stimulated during the refractory period of the represented cellular population.

To translate the output of the oscillator into a transmembrane voltage, a static non-linearity is used. In the case of the MCO, this has been implemented either using Chebyshev polynomials, or more recently (and efficiently) as a look-up table [117]. In the latter case, the output of each $\mathrm{MCO}, y$, is given by:

$$
y=y_{0}+\alpha W(\phi)
$$

where $y_{0}$ is a bias voltage representing the average potential of a cellular assembly and $W($.$) is its$ intrinsic waveform. The waveform is obtained from experimental measurements and is normalized over a phase interval from 0 to 2 pi. 


\subsection{The Cognitive Rhythm Generator (CRG)}

As Dr. Zalay (the creator of the CRG) describes, the Cognitive Rhythm Generator is a hybrid construct that combines the advantages of non-parametric and parametric modeling [103]. It maintains the parametric approach of the MCO and is thus well-suited for modeling mesoscale endogenous electrical phenomena, such as the generation of intrinsic rhythms. Simultaneously however, its use of a non-parametric kernel approach in the form of Principal Dynamic Modes (PDMs) allows it to simply and elegantly capture the complexity of neuronal or cellular coding mechanisms without specific knowledge of the internal physiological structure. In this fashion, it overcomes the deficiencies of either modeling approach, making it well-suited for representing excitable cellular populations.

Shown in Fig. 2.1, the CRG is a mathematical construct consisting of three stages: 1) a bank of PDMs that process and transform inputs, 2) a ring device that is driven by the output of the modes and generates instantaneous phase and amplitude values, and 3) a static non-linearity mapper that maps these values to generate an observable voltage output. Based on this description, it is clear that the major novelty of the CRG as compared to the MCO is the addition of the PDMs. These PDMs give the oscillator its "cognitive" abilities by allowing the modeled population to sense and perceive its environment and to correspondingly modulate its own activity.

Any input, $x_{j}$, to a CRG is processed through convolution with the $k$ th PDM, $m_{k}$, according to:

$$
\varphi_{k, j}(t)=\int_{0}^{\infty} m_{k}(\tau) \cdot x_{j}(t-\tau) d \tau .
$$

These modes are obtained from an eigen-decomposition of measured system Volterra kernels, and provide the CRG with the ability to perform non-linear coding operations observed in the biological system [118] [108]. The modes differ in shape, and can behave as integrators and/or differentiators, with selectivity for particular frequency bands.

The modal outputs are linearly combined or "mixed" and fed into the ring device and mapper to control the activity of the CRG. The stimulus functions $S_{\phi}$ and $S_{\alpha}$ control the phase $(\phi)$ and amplitude $(\alpha)$ of the ring device oscillations, respectively, whereas $S_{y}$ contributes to the observable voltage output in the mapper. These functions take the following form: 


$$
S_{u}=\epsilon_{u} \sum_{k} \sum_{j} \mu_{k, j} \varphi_{k, j}
$$

where $\mu_{k, j}$ and $\epsilon_{u}$ are gains associated with the modal output and the particular stimulus function for $\mathrm{u}=\{\alpha, \phi, \mathrm{y}\}$.

Depending on the type of cell and behavior being modeled, the oscillator ring device may be a clock or a labile clock. As shown in Fig. 2.2, the clock generates an omnipresent rhythm, even in the absence of an input, whereas the labile clock is only active when the input is above a set threshold. The former is more appropriate for cells that exhibit constant rhythmicity, whereas the latter better describes populations that require excitation from other cells to generate an observable voltage membrane rhythm. As such, the clock and labile clock share the same equation for the rate of change of the phase, but differ in the rate of change of the amplitude. The phase dynamics of both ring devices can be modelled by:

$$
\frac{d \phi}{d t}=\omega\left(1+R_{\phi}(\phi) S_{\phi}\right)
$$

where $\omega$ is the oscillator's intrinsic frequency $(\mathrm{rad} / \mathrm{s})$ and $R_{\phi}(\phi)$ is a phase refractory function that prevents the ring from oscillating during the refractory period of the modelled population. The amplitude dynamics of the clock and the labile clock can be described by equations 2.11 and 2.12, respectively:

$$
\begin{gathered}
\frac{d \alpha}{d t}=c_{k} \alpha\left(1+S_{\alpha}-\alpha^{2}\right) \\
\frac{d \alpha}{d t}=c_{k} \alpha^{\frac{1}{3}}\left(V\left(S_{\alpha}\right)-\alpha\right)
\end{gathered}
$$

where $c_{k}$ is a rate constant $(1 / \mathrm{s})$ and $V($.$) is a threshold function that ensures the input to the labile$ clock is supra-threshold. $R_{\phi}($.$) and V($.$) are sigmoidal functions of the form:$

$$
s(u)=\frac{1}{1+\exp \left(-c_{1}\left(u-c_{2}\right)\right)}
$$


where $c_{1}$ and $c_{2}$ are function-dependent constants.

The instantaneous phase and amplitude values are then used by the mapper to generate a realistic voltage output, $y$, according to:

$$
y=S_{y}+\alpha W(\phi)
$$

where $W($.$) is the intrinsic waveform. This waveform is specific to the CRG population type, and$ can be pre-recorded experimentally or synthesized. It is implemented as a look-up table normalized over the phase interval $(0,2 \mathrm{pi}]$.

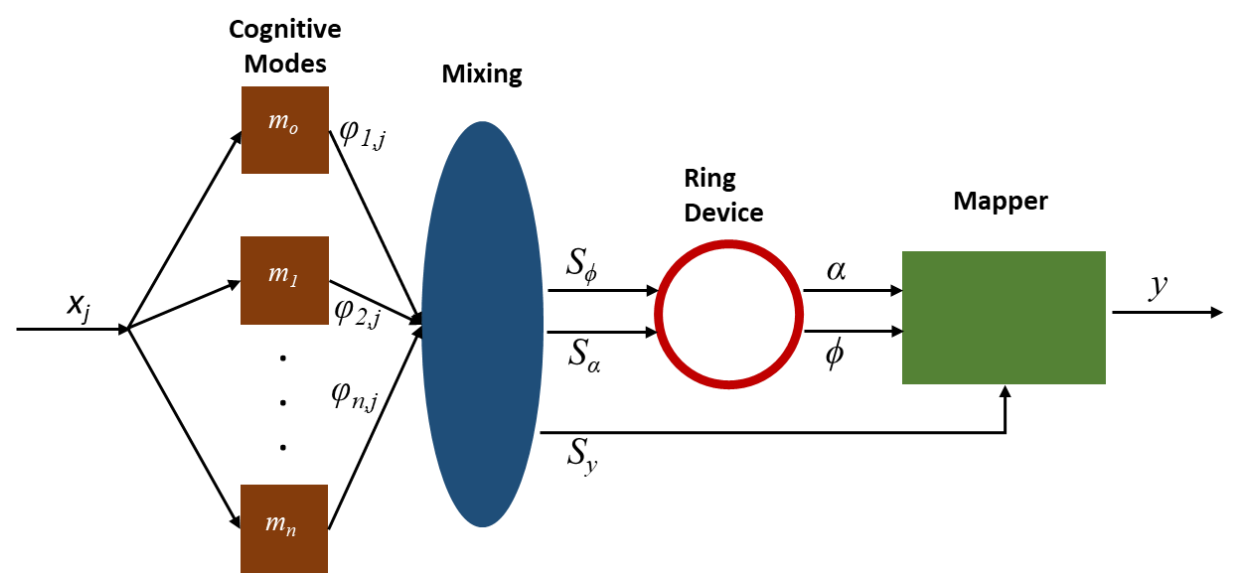

Fig. 2.1. The Cognitive Rhythm Generator (CRG). Any input, $x_{j}$, is processed through $n$ modes, and is transformed into modal outputs $\varphi$. The modal outputs are then linearly combined (mixed) to create stimulus functions to be fed into the ring device and the mapper. The phase stimulus function $\left(\mathrm{S}_{\phi}\right)$ and the amplitude stimulus function $\left(S_{\alpha}\right)$ control the phase and amplitude dynamics of the ring device, respectively. The ring device then generates instantaneous phase $(\phi)$ and amplitude $(\alpha)$ values that are used in a waveform lookup table in the mapper. This is finally combined with the $S_{y}$ stimulus function to produce a transmembrane voltage output, $y$. 
(a)
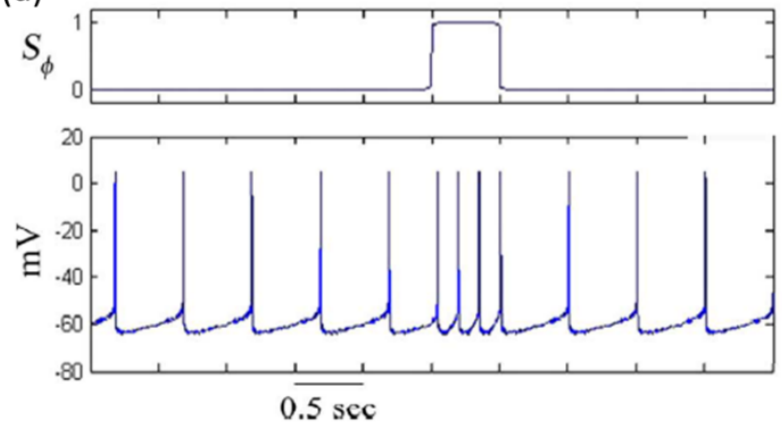

(b)
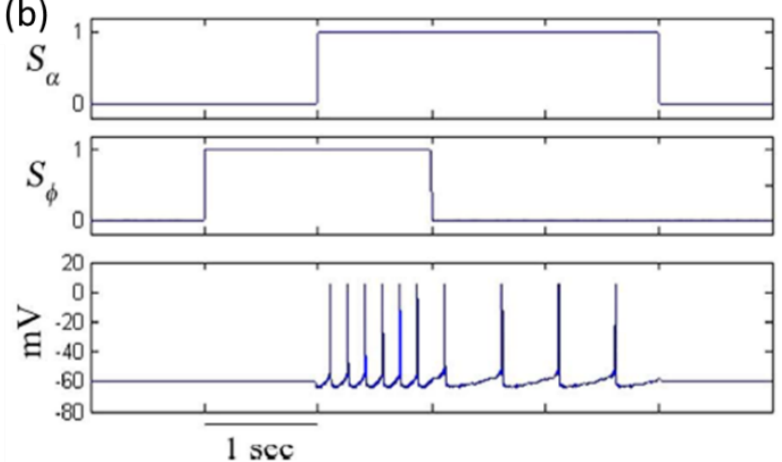

Fig. 2.2. Behavior of the clock and labile clock ring devices (Adapted from [117]). a) The clock generates an intrinsic rhythm in the absence of any stimulus. As it is subjected to a phase stimulus, $S_{\phi}$, however, it changes the frequency of the oscillation. b) The labile clock cannot generate any output in the absence of an amplitude stimulus, $S_{\alpha}$, regardless of the phase stimulus. It responds to the phase stimulus in the same manner as the clock since they share the same equation for the rate of change of phase.

\subsection{SED-Generation using Coupled CRGs}

Very briefly discussed below are two neuronal CRG networks capable of generating SEDs. Details, particularly regarding the mathematics, are omitted and are discussed in Chapter 3 with respect to the neuroglial model developed for this thesis. The HFO SED network and the neuroglial model, developed by Farah et al., are heavily inspired by the original SED network created by Zalay et al. [119].

\subsubsection{The Original SED Network}

Zalay et al. created a network capable of generating SEDs similar to SLEs observed in a low- $\mathrm{Mg}^{2+}$ / High- $\mathrm{K}^{+}$experimental mouse model of epilepsy [119]. The network modeled the circuitry and rhythmicity of the CA3, with two CRGs representing pyramidal neuron populations and two representing interneuron populations (Fig. 2.3a). The pyramidal populations exhibit autoassociative self-feedback and mutual excitatory connections, while the interneurons provided inhibitory feedback.

As can be seen from the simulated extracellular (EC) field potential in Fig. 2.3b, the model is capable of generating spontaneous recurrent SEDs, as a result of the summation of synchronous ictal-like discharges in two or more CRGs. Zalay et al. further demonstrated close resemblance 
between the experimental SLEs and the model-generated SEDs at the cellular and field potential level.

(a)

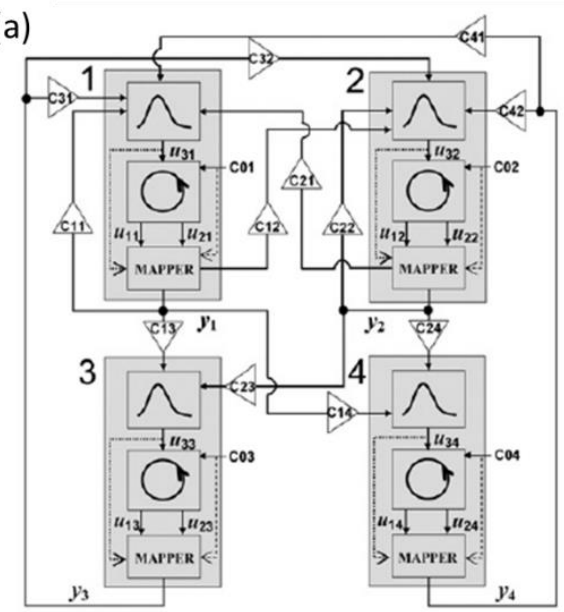

(b)

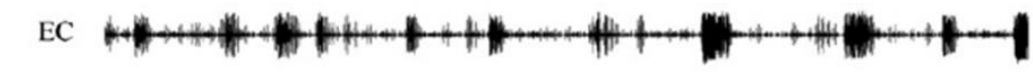

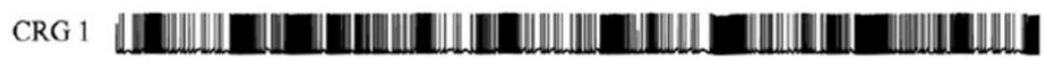

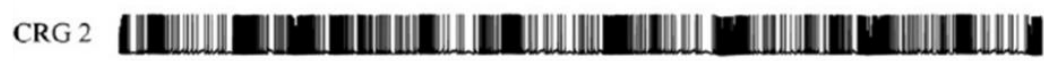

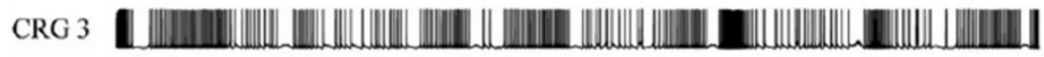

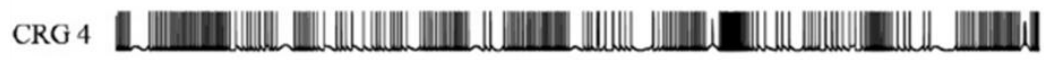

Fig. 2.3. Spontaneous recurrent SEDs in a network of 4 coupled CRGs (Adapted from [119]). a) The network model consists of 4 CRGs, with CRGs 1 and 2 representing pyramidal cell populations and CRGs 3 and 4 representing GABAergic interneurons. There are excitatory connections between the pyramidal populations and from the pyramidal populations to the interneurons. The interneurons provide inhibitory feedback to the pyramidal cells. b) The EC field potential trace exhibits spontaneous recurrent SEDs. During the SEDs, two or more of the CRGs exhibit ictal-like discharges.

\subsubsection{The HFO SED Network}

The HFO SED network, presented at the 2016 Society for Autonomous NeuroDynamics (SAND) conference, aimed to build on the original SED network in two ways [120]. First, the number of CRGs is doubled with the goal being to bring about dynamics that may have been unachievable using a smaller network size. Second and more importantly, oscillators with intrinsic frequencies in the HFO range were added to observe the presence of HFOs, which were absent in the original model.

As shown in Fig. 2.4, the network consists of 4 excitatory pyramidal cell CRGs oscillating in the theta LFO range and 4 inhibitory interneuron CRGs oscillating the ripple HFO range. The pyramidal cell CRGs employ a clock ring device, while interneurons which often require external input to burst, employ a labile clock ring device. As with the original model, there were recurrent 
excitatory connections among the pyramidal CRGs and inhibitory feedback from the interneurons. Furthermore, there were mutual inhibitory connections between the interneurons. These forms of connections are all found in the hippocampus, as discussed in section 1.2.

Shown in Fig. 2.5 are the transmembrane voltage outputs of all 8 CRG units with a simulated Local Field Potential (LFP) at the top. As a result of ictal-like discharges in the individual populations, spontaneous recurrent SEDs arise in the LFP without any form of external input into the model. These SEDs, both at the intracellular and extracellular level, looked similar to the SLEs recorded in the low- $\mathrm{Mg}^{2+} /$ high- $\mathrm{K}^{+}$experimental model of epilepsy (Fig. 2.6). Further analysis of the SEDs revealed evidence of delta-HFO and theta-HFO phase-amplitude CFC.

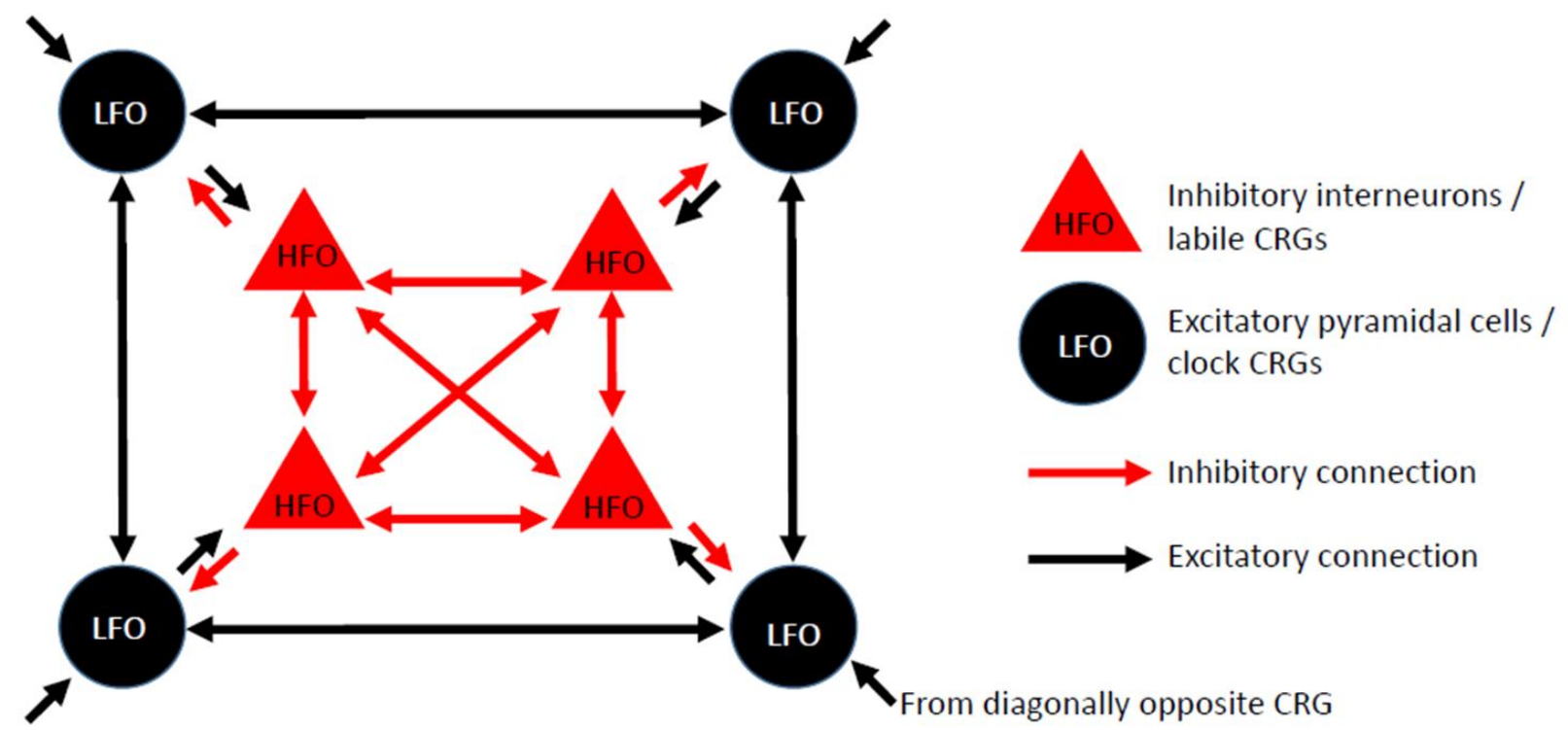

Fig. 2.4. Model schematic of the HFO SED model [120]. The network consists of 8 CRGs, 4 of which represent pyramidal cell populations oscillating in the theta LFO range, and 4 that represent inhibitory interneuron populations oscillating in the ripple HFO range. The pyramidal CRGs use a clock ring device, while the interneurons use a labile clock ring device. There are mutual excitatory connections among pyramidal CRGs, mutual inhibitory connections among interneurons, excitatory connections from the pyramidal cells to the interneurons, and inhibitory feedback connections from the interneurons to the pyramidal cells. 


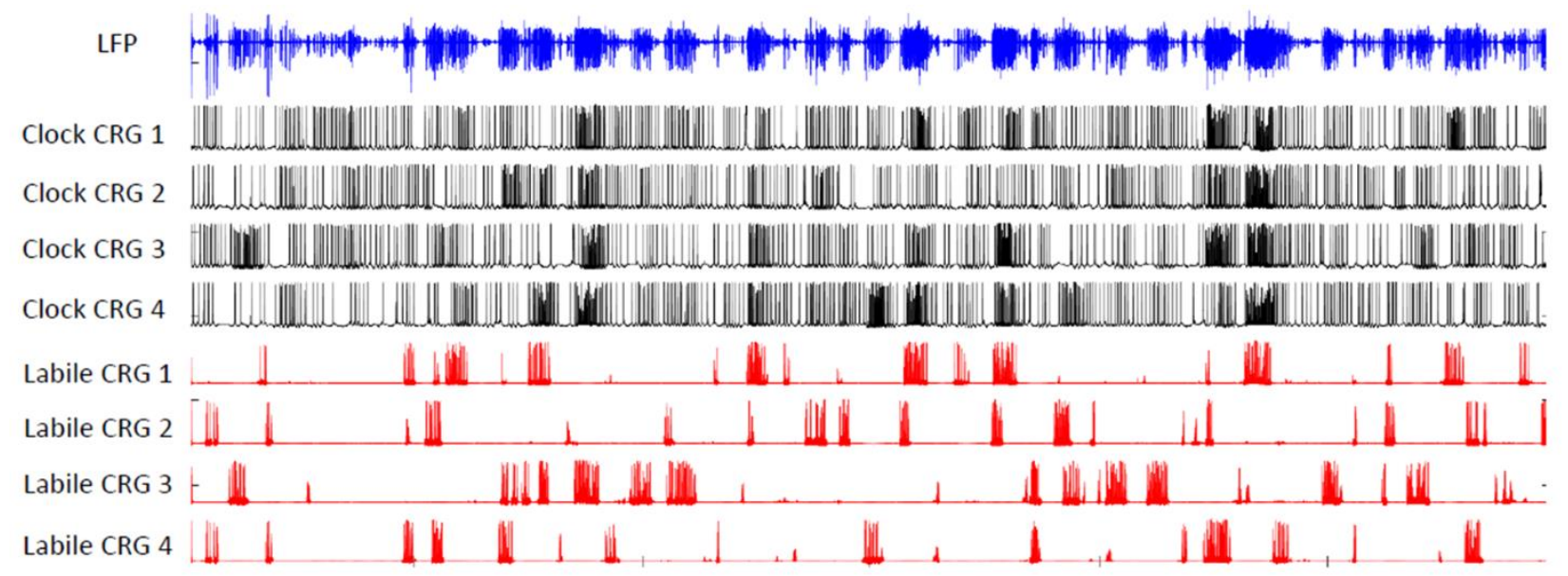

Fig. 2.5. Spontaneous recurrent SEDs as observed from the model outputs [120]. The top trace depicts the LFP, and the remaining lower traces are the membrane voltage $\left(\mathrm{V}_{\mathrm{m}}\right)$ outputs of each of the 8 CRGs. During an SED in the LFP, two or more of the neuronal populations exhibit ictal-like discharges.

(a)

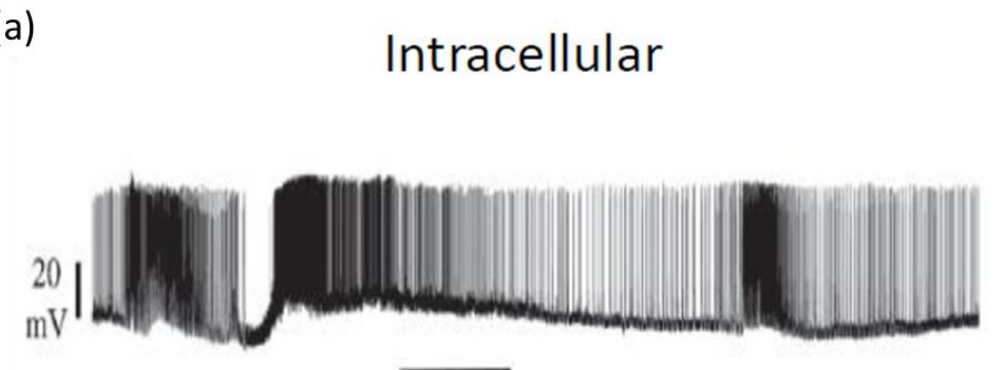

$$
10 \mathrm{~s}
$$

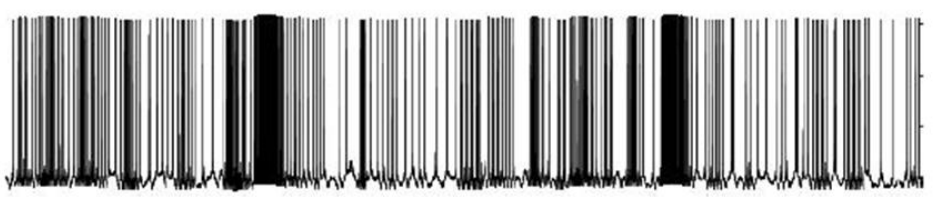

Clock CRG (b)

\section{Extracellular Field}
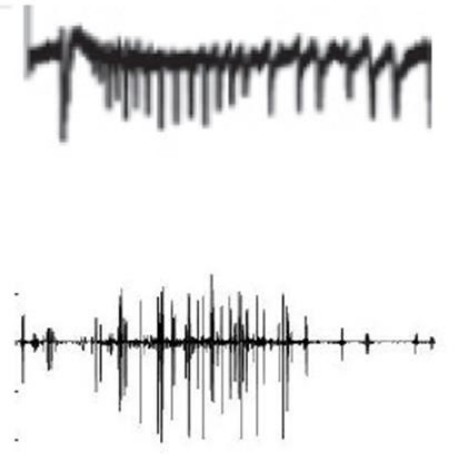

Model LFP

Fig. 2.6. Comparison of the HFO SED model outputs with recordings from the low-Mg' ${ }^{2+}$ high-K ${ }^{+}$ experimental model of epilepsy [120]. a) The transmembrane voltage output of a clock pyramidal CRG (bottom) looks similar to the intracellular recordings from pyramidal neurons (top). b) The simulated LFP of an SED (bottom) resembles the experimental extracellular field recordings (top). The recordings used in this figure are adapted from [119]. 


\section{Chapter 3}

\section{Model and Methods}

\subsection{The Neuroglial Network Model}

The neuroglial network model, shown in Fig. 3.1, contains 16 CRGs organized into four subunits. A subunit contains four CRGs, each of which is used to model one of the following populations: a pyramidal cell population, an interneuron population, a microglial population, and an astrocyte population. The pyramidal CRGs are numbered 1 to 4 , the interneurons 5 to 8 , the microglia 9 to 12 , and the astrocytes 13 to 16.

\subsubsection{Mathematics of the Network Model}

The general CRG, described in Chapter 2, is transformed to Cartesian form and adapted for specific use in the neuroglial network model. The polar variables, $\alpha$ and $\phi$, can be transformed to Cartesian state variables $\mathrm{u}_{1}$ and $\mathrm{u}_{2}$ according to the following equations:

$$
\begin{aligned}
& u_{1}=\alpha \sin \phi \\
& u_{2}=\alpha \cos \phi
\end{aligned}
$$

In Cartesian form, each ring device of the $n$th CRG can then be represented by a system of two equations. Equations (3.3) and (3.4) are for the clock and labile clock, respectively:

$$
\begin{gathered}
\dot{u}_{1 n}=\omega_{n}\left[u_{2 n}\left(1+R_{\phi, n}\left(\tan ^{-1} \frac{u_{2 n}}{u_{1 n}}\right) S_{\phi, n}\right)+u_{1 n}\left(1+S_{\alpha, n}-u_{1 n}^{2}-u_{2 n}^{2}\right)\right] \\
\dot{u}_{2 n}=\omega_{n}\left[-u_{1 n}\left(1+R_{\phi, n}\left(\tan ^{-1} \frac{u_{2 n}}{u_{1 n}}\right) S_{\phi, n}\right)+u_{2 n}\left(1+S_{\alpha, n}-u_{1 n}^{2}-u_{2 n}^{2}\right)\right] \\
\dot{u}_{1 n}=\omega_{n} u_{2 n}\left(1+R_{\phi, n}\left(\tan ^{-1} \frac{u_{2 n}}{u_{1 n}}\right) S_{\phi, n}\right)+c_{k} u_{1 n}\left(u_{1 n}^{2}+u_{2 n}^{2}\right)^{-\frac{1}{3}}\left(V_{n}\left(S_{\alpha, n}\right)-\left(u_{1 n}^{2}+u_{2 n}{ }^{2}\right)^{\frac{1}{2}}\right) \\
\dot{u}_{2 n}=-\omega_{n} u_{1 n}\left(1+R_{\phi, n}\left(\tan ^{-1} \frac{u_{2 n}}{u_{1 n}}\right) S_{\phi, n}\right)+c_{k} u_{2 n}\left(u_{1 n}^{2}+u_{2 n}^{2}\right)^{-\frac{1}{3}}\left(V_{n}\left(S_{\alpha, n}\right)-\left(u_{1 n}{ }^{2}+u_{2 n}{ }^{2}\right)^{\frac{1}{2}}\right)
\end{gathered}
$$


where $\omega_{n}$ is the intrinsic frequency of the oscillator, $R_{\phi, n}($.$) is the phase refractory function, \tan ^{-1}$ is the 4-quadrant arctangent function used to obtain the phase angle, $c_{k}$ is a rate constant, $S_{\phi, n}$ and $S_{\alpha, n}$ are the stimulus functions, and $V_{n}($.$) is the labile clock threshold function.$

Regarding the PDMs, only the integrating mode is used. The choice to omit other higher-order modes was made for two reasons. First, in experimental measurements from hippocampal neurons, the integrating mode dominated in terms of the relative contribution to the overall system response [121]. Second, by including only the integrating mode, it is possible to model the convolution operation by a system of first-order differential equations, rather than integro-differential equations [102].

The integrating mode, $m(t)$, can be represented by an exponential impulse response function [119] [102]:

$$
m(t)=\beta t \exp (-\beta t)
$$

where $1 / \beta$ is the modal time constant controlling the decay rate of the mode response and thus the extent of integration. $\beta=2$ was measured in hyperexcitable conditions in a low- $\mathrm{Mg}^{2+} / \mathrm{high}^{+} \mathrm{K}^{+}$ experimental model of epilepsy, and this value was used for all computations in this thesis. Using Laplace Transforms, the convolution described in Equation (2.8) becomes [102]:

$$
\begin{gathered}
\dot{u}_{3 n}=u_{4 n} \\
\dot{u}_{4 n}=\beta f_{n}-2 \beta u_{4 n}-\beta^{2} u_{3 n}
\end{gathered}
$$

where $u_{3 n}$ and $u_{4 n}$ are state variables representing the amplitude of the mode output and its rate of change respectively, and $f_{n}$ is the input to the $\mathrm{CRG}$.

The output of the integrating mode is fed through the stimulus functions into the ring device and the mapper. In this model, the stimulus functions are defined as follows:

$$
\begin{aligned}
& S_{\phi, n}=\varepsilon_{\phi, n}\left(c_{0 n}+k_{n} u_{3 n}\right) \\
& S_{\alpha, n}=\varepsilon_{\alpha, n}\left(c_{0 n}+k_{n} u_{3 n}\right) \\
& S_{y, n}=\varepsilon_{y, n}\left(c_{0 n}+\eta_{n} u_{3 n}\right)
\end{aligned}
$$


where $\varepsilon_{\phi, n}, \varepsilon_{\alpha, n}, \varepsilon_{y, n}, k_{n}$, and $\eta_{n}$ are gains, and $c_{0 n}$ is a constant offset. In the case of the clocks, which do not have a threshold function, the $S_{\phi}$ stimulus function is rectified at -1 to ensure zero or positive phase advance.

The state variables obtained from solving a system of 4 differential equations for each CRG are then used in the final mapper stage to obtain the observable output, $y_{n}$ :

$$
y_{n}=S_{y, n}+\left(u_{1 n}{ }^{2}+u_{2 n}{ }^{2}\right)^{\frac{1}{2}} W\left(\tan ^{-1} \frac{u_{2 n}}{u_{1 n}}\right)
$$

The CRGs are coupled to one another, such that the input, $f_{n}$, to the nth CRG is a linear combination of the observable outputs of M CRG units, weighted by coupling coefficients according to:

$$
f_{n}=\sum_{m=1}^{M} c_{m: n} y_{m}+x_{n}
$$

where $c_{m: n}$ is the coupling coefficient from CRG $m$ to CRG $n, y_{m}$ is the output of the mth CRG, and $x_{n}$ is an optional external input .

A Local Field Potential (LFP) at the center of the network is simulated to obtain a collective overview of the activity of all 16 CRGs [122]. Treating the CRGs as point sources, the LFP, $\psi$, can be approximated by:

$$
\psi=\sum_{n}^{16} \lambda_{n} \frac{d^{2} y_{n}}{d t^{2}}
$$

where $\lambda_{n}$ is a constant that takes into account the distance between the $n$th cellular assembly and the recording electrode, as well as system properties, such as the conductivity [117]. 


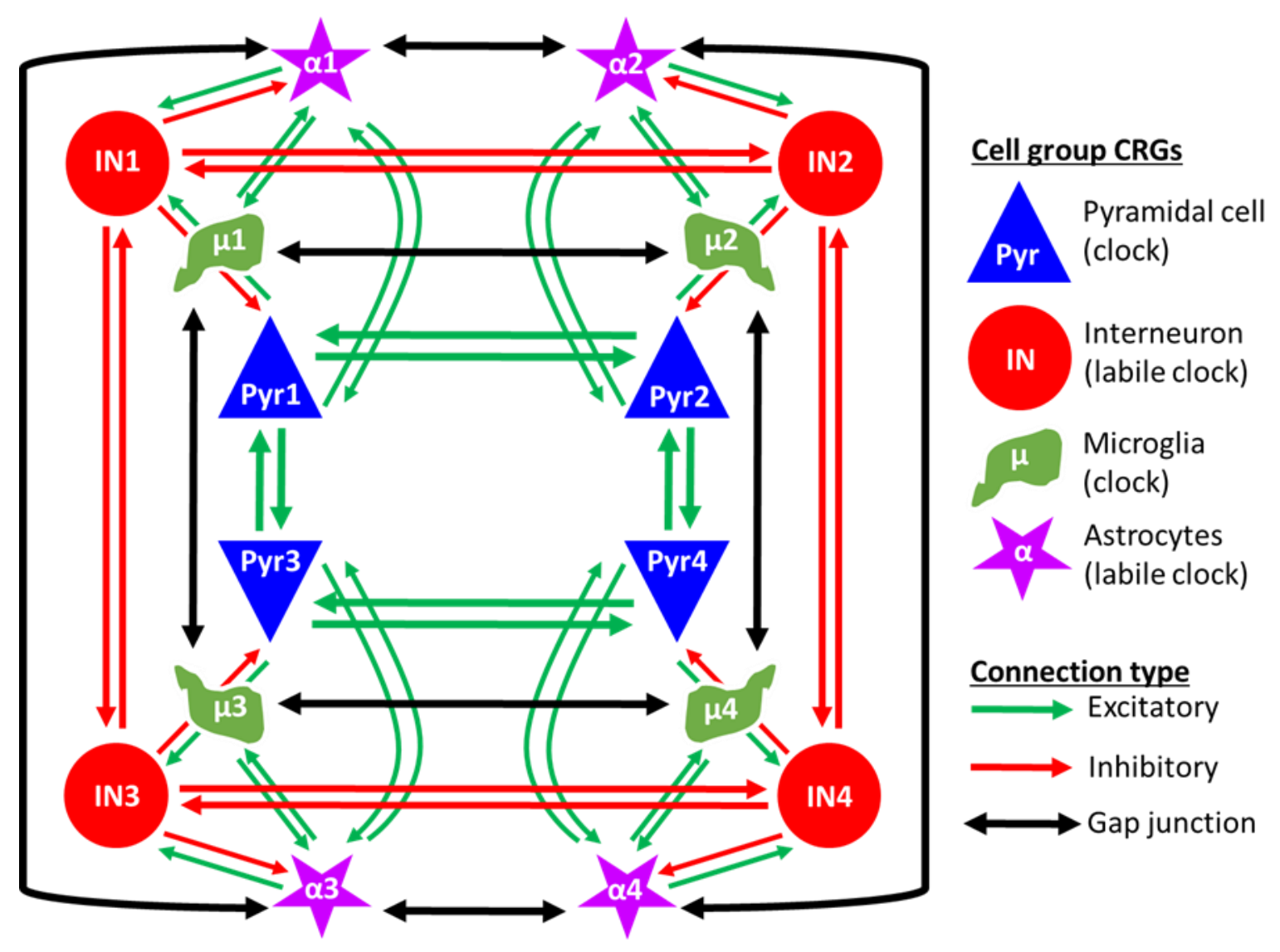

Fig. 3.1. The CRG neuroglial network model. 16 coupled CRGs are arranged into four subunits, each containing a population of pyramidal cells, interneurons, microglia, and astrocytes, with intrinsic frequencies in the theta, HFO, low delta, and delta frequencies, respectively. The pyramidal and microglial CRGs use clock ring devices, while the interneuron and astrocyte CRGs use labile clocks. Within each subunit, there are excitatory connections from the pyramidal populations and the astrocytes to all other CRGs, and inhibitory connections from the interneurons to all other CRGs. The microglial CRGs have an inhibitory "pruning" role on the neuronal synapses, but an excitatory effect on the astrocytes. Between subunits, the pyramidal populations are connected by excitatory connections, the interneurons by inhibitory connections, and the microglia and astrocytes by gap junctions. Although the subunits are identical in layout, they differ from one another in the intrinsic frequencies of the contained CRGs.

\subsubsection{Connectivity and Features of the Network Model}

The pyramidal cell CRGs exhibit constant rhythmicity and employ a clock ring device with intrinsic frequencies in the theta range. They are excitatory, and have auto-associative selffeedback, as well as mutual connections among one another. Each population of pyramidal cells is connected to a population of interneurons. The interneuron CRGs contain a labile ring device, and thus can only become active when the total input is supra-threshold. They are the source of 
HFOs, oscillating with intrinsic frequencies in the ripple range. The interneuron CRGs are inhibitory, acting as a source of GABAergic connectivity. They perform feedback inhibition on the pyramidal populations, as well as mutual inhibition among one other.

The microglial CRGs use a clock ring device and have low-delta intrinsic frequencies, ranging from $0.2 \mathrm{~Hz}$ to $0.5 \mathrm{~Hz}$. They are connected to one another via gap junctions, and interact directly with the astrocytes through excitatory connections. The astrocytes are modelled to be labile, with intrinsic frequencies in the delta range. They too interact with one another via gap junctions, and have positive feedback connections with the microglia. Gap junctions in the model are symmetrically bidirectional connections, where the coupling coefficients between two connected groups are equal in both directions.

In addition to intra-neuron and intra-glial connections, there are also connections between neuronal and glial populations. The pyramidal CRGs maintain their excitatory nature in their connections with both astrocytes and microglia, whereas the interneuron CRGs are connected to the glia via inhibitory coupling coefficients. The microglial connection to the neurons is inhibitory, acting through a synaptic "pruning" mechanism. Based on their level of activation, the microglia can reduce the coupling between neurons according to:

$$
\rho=1-c_{m: n} P\left(V_{\mu}\right)
$$

where $\rho$ is a value ranging from 0 and 1 that is multiplied by the intra-neuron coupling coefficients, $\mathrm{c}_{\mathrm{m}: \mathrm{n}}$ is the coupling coefficient from microglial CRG $m$ to neuronal CRG $n$, and $P(V \mu)$ is a sigmoidal pruning function acting on the scaled output of the microglial membrane voltage and taking the form of Equation (2.13). This pruning mechanisms is described in more detail in the Appendix. Finally, the astrocytes are connected to both neuronal populations by excitatory connections, representing a variety of astrocyte-neuron communication mechanisms, such as the release of glutamate and D-Serine.

All model coupling coefficients and parameters are presented in Table 3.1 and Table 3.2, respectively. 
TABLE 3.1

NEUROGLIAL NETWORK MODEL COUPLING COEFFICIENTS

\begin{tabular}{|c|c|c|}
\hline Connection Type & Coupling Coefficients & Value \\
\hline Intra-Pyramidal & $\begin{array}{l}\mathrm{c}_{1: 1}, \mathrm{c}_{2: 2}, \mathrm{c}_{3: 3}, \mathrm{c}_{4: 4}, \mathrm{c}_{1: 2,}, \mathrm{c}_{1: 3} \\
\mathrm{c}_{2: 1}, \mathrm{c}_{2: 4}, \mathrm{c}_{3: 1}, \mathrm{c}_{3: 4}, \mathrm{c}_{4: 2}, \mathrm{c}_{4: 3}\end{array}$ & 0.1 \\
\hline Intra-Interneuron & $\begin{array}{c}\mathrm{c}_{5: 6}, \mathrm{c}_{5: 7}, \mathrm{c}_{6: 5}, \mathrm{c}_{6: 8}, \mathrm{c}_{7: 5}, \mathrm{c}_{7: 8} \\
\mathrm{c}_{8: 6,6}, \mathrm{c}_{8: 8}\end{array}$ & -0.7 \\
\hline Intra-Micro & $\begin{array}{c}\mathrm{C}_{9: 10}, \mathrm{C}_{9: 11}, \mathrm{C}_{10: 9}, \mathrm{C}_{10: 12}, \mathrm{C}_{11: 9} \\
\mathrm{c}_{11: 12}, \mathrm{C}_{12: 10}, \mathrm{C}_{12: 11}\end{array}$ & 0.2 \\
\hline Intra-Astro & $\begin{array}{l}\mathrm{c}_{13: 14}, \mathrm{c}_{13: 15}, \mathrm{c}_{14: 13}, \mathrm{c}_{14: 16} \\
\mathrm{c}_{15: 13}, \mathrm{c}_{15: 16}, \mathrm{c}_{16: 14}, \mathrm{c}_{16: 15}\end{array}$ & 0.2 \\
\hline Pyramidal-Interneuron & $\mathrm{c}_{1: 5,}, \mathrm{c}_{2: 6}, \mathrm{c}_{3: 7}, \mathrm{c}_{4: 8}$ & 0.6 \\
\hline Interneuron-Pyramidal & $\mathrm{c}_{5: 1}, \mathrm{c}_{6: 2,}, \mathrm{c}_{7: 3}, \mathrm{c}_{8: 4}$ & -0.65 \\
\hline Micro-Astro & $\mathrm{c}_{9: 13}, \mathrm{c}_{10: 14,} \mathrm{c}_{11: 15}, \mathrm{c}_{12: 16}$ & 1 \\
\hline Astro-Micro & $\mathrm{c}_{13: 9}, \mathrm{c}_{14: 10}, \mathrm{c}_{15: 11,} \mathrm{c}_{16: 12}$ & 0.1 \\
\hline Pyramidal-Micro & $\mathrm{c}_{1: 9}, \mathrm{c}_{2: 10}, \mathrm{c}_{3: 11}, \mathrm{c}_{4: 12}$ & 0.2 \\
\hline Micro-Pyramidal & $\mathrm{c}_{9: 1}, \mathrm{c}_{10: 2}, \mathrm{c}_{11: 3}, \mathrm{c}_{12: 4}$ & 1 \\
\hline Interneuron-Micro & $\mathrm{c}_{5: 9}, \mathrm{c}_{6: 10}, \mathrm{c}_{7: 11,}, \mathrm{c}_{8: 12}$ & -0.6 \\
\hline Micro-Interneuron & $\mathrm{c}_{9: 5}, \mathrm{c}_{10: 6}, \mathrm{c}_{11: 7}, \mathrm{c}_{12: 8}$ & 1 \\
\hline Pyramidal-Astro & $\mathrm{c}_{1: 13}, \mathrm{c}_{2: 14}, \mathrm{c}_{3: 15}, \mathrm{c}_{4: 16}$ & 0.8 \\
\hline Astro-Pyramidal & $\mathrm{c}_{13: 1}, \mathrm{c}_{14: 2}, \mathrm{c}_{15: 3}, \mathrm{c}_{16: 4}$ & 0.3 \\
\hline Interneuron-Astro & $\mathrm{c}_{5: 13,}, \mathrm{c}_{6: 14}, \mathrm{c}_{7: 15}, \mathrm{c}_{8: 16}$ & -0.2 \\
\hline Astro-Interneuron & $\mathrm{c}_{13: 5,} \mathrm{c}_{14: 6}, \mathrm{c}_{15: 7}, \mathrm{c}_{16: 8}$ & 0.2 \\
\hline
\end{tabular}

TABLE 3.2

NEUROGLIAL NETWORK MODEL PARAMETERS

\begin{tabular}{|c|c|c|c|c|c|c|c|c|c|c|c|c|c|c|c|c|c|}
\hline \multirow[b]{2}{*}{ Parameter Name } & \multirow[b]{2}{*}{$\begin{array}{c}\text { Parameter } \\
\text { symbol(s) }\end{array}$} & \multicolumn{4}{|c|}{ Pyramidal CRGs } & \multicolumn{4}{|c|}{ Interneuron CRGs } & \multicolumn{4}{|c|}{ Microglial CRGs } & \multicolumn{4}{|c|}{ Astrocyte CRGs } \\
\hline & & 1 & 2 & 3 & 4 & 5 & 6 & 7 & 8 & 9 & 10 & 11 & 12 & 13 & 14 & 15 & 16 \\
\hline Intrinsic Frequency & $\omega_{\mathrm{n}}$ & $8 \pi$ & $8.4 \pi$ & $8.8 \pi$ & $9.2 \pi$ & $200 \pi$ & $210 \pi$ & $220 \pi$ & $230 \pi$ & $0.4 \pi$ & $0.6 \pi$ & $0.8 \pi$ & $1 \pi$ & $2 \pi$ & $2.4 \pi$ & $2.8 \pi$ & $3.2 \pi$ \\
\hline Constant offset & $c_{0 n}$ & 0 & \begin{tabular}{|l|}
-0.2 \\
\end{tabular} & -0.2 & -0.2 & 0 & 0 & 0 & 0 & -0.2 & -0.2 & \begin{tabular}{|l|}
-0.3 \\
\end{tabular} & 0 & \begin{tabular}{|l|}
-0.3 \\
\end{tabular} & -0.3 & \begin{tabular}{|l|}
-0.3 \\
\end{tabular} & -0.3 \\
\hline \multirow{5}{*}{ Stimulus Gain } & $\boldsymbol{\epsilon}_{\phi, \mathbf{n}}$ & 1 & 1 & 1 & 1 & 1 & 1 & 1 & 1 & 1 & 1 & 1 & 1 & 0.3 & 0.3 & 0.4 & 0.4 \\
\hline & $\epsilon_{\alpha, \mathrm{n}}$ & 0 & 0 & 0 & 0 & 1 & 1 & 1 & 1 & 0 & 0 & 0 & 0 & 1 & 1 & 1 & 1 \\
\hline & $\epsilon_{\mathrm{y}, \mathrm{n}}$ & 1 & 1 & 1 & 1 & 1 & 1 & 1 & 1 & 1 & 1 & 1 & 1 & 1 & 1 & 1 & 1 \\
\hline & $k_{n}$ & 10 & 10 & 10 & 10 & 1 & 1 & 1 & 1 & 10 & 10 & 10 & 10 & 10 & 10 & 10 & 10 \\
\hline & $\eta_{n}$ & 1 & 1 & 1 & 1 & 1 & 1 & 1 & 1 & 1 & 1 & 1 & 1 & 0 & 0 & 0 & 0 \\
\hline Rate constant & $c_{k}$ & & & & & 1000 & 1000 & 1000 & 1000 & & & & & $2 \pi$ & $2.4 \pi$ & $2.8 \pi$ & $3.2 \pi$ \\
\hline \multirow{2}{*}{$\begin{array}{l}\text { Refractory Function } \\
(\mathrm{R} \phi)\end{array}$} & c1 & 1 & 1 & 1 & 1 & 25 & 25 & 25 & 25 & 1 & 1 & 1 & 1 & 1 & 1 & 1 & 1 \\
\hline & c2 & $-\infty$ & $-\infty$ & $-\infty$ & $-\infty$ & 0.7 & 0.7 & 0.7 & 0.7 & $-\infty$ & $-\infty$ & $-\infty$ & $-\infty$ & $-\infty$ & $-\infty$ & $-\infty$ & $-\infty$ \\
\hline \multirow{2}{*}{$\begin{array}{l}\text { Threshold function } \\
\text { (V) }\end{array}$} & c1 & & & & & 50 & 50 & 50 & 50 & & & & & 1000 & 1000 & 1000 & 1000 \\
\hline & c2 & & & & & 0.25 & 0.25 & 0.25 & 0.25 & & & & & -0.10 & -0.04 & -0.17 & -0.20 \\
\hline \multirow{2}{*}{$\begin{array}{l}\text { Pruning function } \\
\text { (P) }\end{array}$} & c1 & & & & & & & & & 1 & 1 & 1 & 1 & & & & \\
\hline & c2 & & & & & & & & & 8 & 8 & 8 & 8 & & & & \\
\hline LFP population gain & $\lambda_{\mathrm{n}}$ & 1 & 1 & 1 & 1 & 1 & 1 & 1 & 1 & 1 & 1 & 1 & 1 & 1 & 1 & 1 & 1 \\
\hline
\end{tabular}




\subsubsection{The Solver}

The system of nonlinear ordinary differential equations were integrated using a stiff differential equation solver. While stiffness is a difficult concept to define, MathWorks describes it in the following way [123]:

"An ordinary differential equation problem is stiff if the solution being sought is varying slowly, but there are nearby solutions that vary rapidly, so the numerical method must take small steps to obtain satisfactory results."

As such, ode15s (stiff/NDF), a multistep solver that uses variable-order numerical differentiation formulas, was used. All simulations were performed in Simulink.

\subsection{Analysis Methods}

The following 3 sections describe the main techniques used for the analysis of the model results.

\subsubsection{State Duration Statistics}

To investigate state duration statistics and transitions, the durations of the SEDs and the time between them (inter-SED durations) were first obtained using an automated detection system, similar to that used in [124] [125]. The LFP signal was squared and convolved with a Gaussian kernel of 2000-point aperture to obtain an envelope. This envelope was then normalized and a threshold was used to separate SED from non-SED states.

To gain a better understanding of the underlying distributions of the model-generated SEDs, a Gamma Mixture Model (GMM) was applied. Using the algorithm and code provided in [126], two components are estimated using the EM algorithm. The mixture density function, $g($.$) , is given by:$

$$
g(x)=p f_{1}(x)+(1-p) f_{2}(x)
$$

where $p$ and 1-p are the mixing proportions, and $f_{1}($.$) and f_{2}($.$) are the gamma component densities.$

Based on the GMM results and a 2 second threshold observed in biological systems [92] [127], the SED durations were then split into short duration SEDs (sSEDs) if shorter than 2 seconds and long duration SEDs (ISEDs) if longer than 2 seconds. From the non-SED states, the durations 
between consecutive sSEDs (inter-sSEDs) and the durations between consecutive 1SEDs (inter1SEDs) were extracted.

The durations of the inter-sSEDs, sSEDs, inter-1SEDs, and ISEDs were fitted with gamma distributions of the following form:

$$
y=C(x-\delta)^{\alpha-1} \exp \left(-\frac{x-\delta}{\beta}\right)
$$

where $C$ is a normalization constant, $\alpha$ is the shape parameter, $\beta$ is the scale parameter, and $\delta$ is a shift in the distribution. $\alpha$ is of interest as the shape of the distribution can be indicative of a random or deterministic process [125] [124]. In the case that $\alpha<1$, the distribution is monotonically decreasing with a maximum at the origin, indicating a random-walk type process. In the case of $\alpha$ $=1$, the function reduces to a decaying exponential distribution, indicating a Poisson-type process. If $\alpha>1$, the distribution has a non-zero maximum and is indicative of a deterministic state termination process. The maximum likelihood estimates with $95 \%$ confidence intervals were obtained using the gamfit function in MATLAB R2016a.

\subsubsection{Quantifying the Excitability Level of the System}

In order to assess the extent of the system's hyperexcitability under a specific set of conditions or parameters, a measure of excitation level must be used. The excitation function (Fig. 3.2), E(t), introduced in [102] [103], is given by:

$$
E(t)=\sum_{n=1}^{16} u_{3 n}(t)^{2}
$$

where $u_{3}$ is the integrating mode response amplitude described in section 3.1.1. The integrating mode responses are directly correlated with network excitability. The overall excitability level of the system was then quantified by taking the integral of the excitation function using the trapezoidal rule. Zalay has previously demonstrated that this function correlates well with other energy measures, such as the Teager energy operator [103]. 
(a)

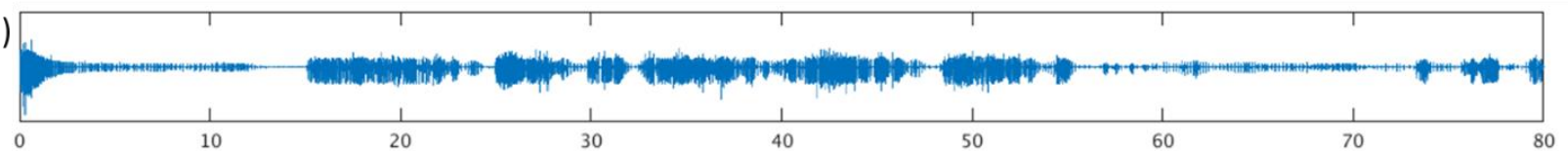

(b)

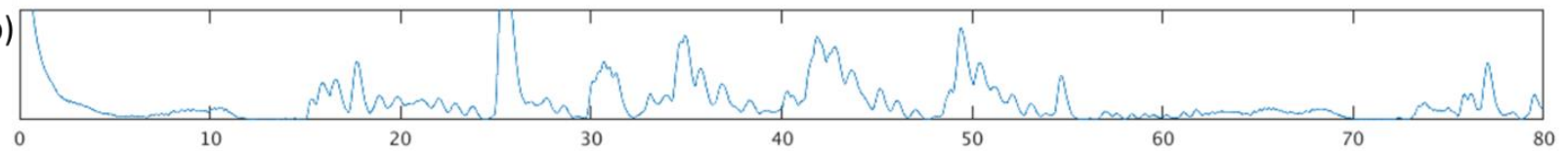

Fig. 3.2. The excitation function. a) A simulated LFP with SEDs of various durations. b) The corresponding excitability function. Note how rises in the excitability function correspond well with the occurrence of model SEDs.

\subsubsection{LFO-HFO Phase-Amplitude Coupling}

To study the cross-frequency interactions within an SED, the Index of Cross Frequency Coupling ( $\mathrm{I}_{\mathrm{cfc}}$ ) was computed on the LFP signal. This method, developed by Tort et al., measures the extent of phase-amplitude coupling, particularly how the amplitude of high frequency rhythms is modulated by the phase of low frequency rhythms [91].

The steps of the algorithm are described in more detail in [33] [91]. The phase time series of the LFO rhythms $\left(\varphi_{L}(t)\right)$ and the envelope of the amplitude time series of the HFO rhythms $\left(A_{H}(t)\right)$ were extracted from wavelet coefficients. To obtain the wavelet coefficients, a Morlet mother wavelet with a center frequency of $0.8125 \mathrm{~Hz}$ and a bandwidth of $5 \mathrm{~Hz}$ was used. The phases were binned into 18 phase bins at $20^{\circ}$ increments, and the mean of the envelope amplitudes were computed over each bin. The amplitude envelope mean at each bin was then normalized with respect to the sum of all amplitude envelopes at all 18 bins, to obtain an amplitude distribution. In the case that there is no phase-amplitude coupling, this distribution is uniform. As such, the amplitude distribution is compared to a uniform distribution by computing the Kullback-Leibler (KL) distance between them. A larger distance indicates stronger phase-amplitude coupling and a higher value for the $\mathrm{I}_{\mathrm{cfc}}$. The result of the algorithm is a two-dimensional comodulogram matrix that can be visualized by mapping the $\mathrm{I}_{\mathrm{cfc}}$ value to a color. An example is shown in Fig. 3.3. 
(a)

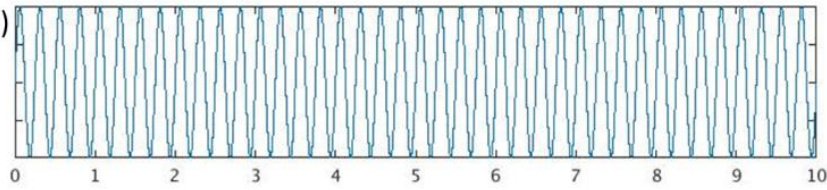

(b)

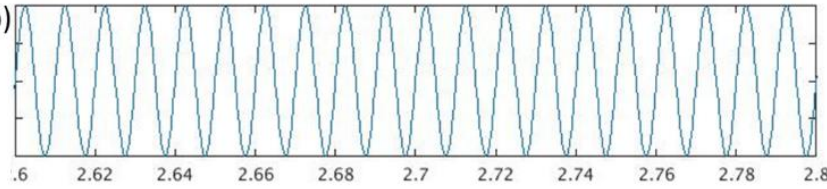

(c)

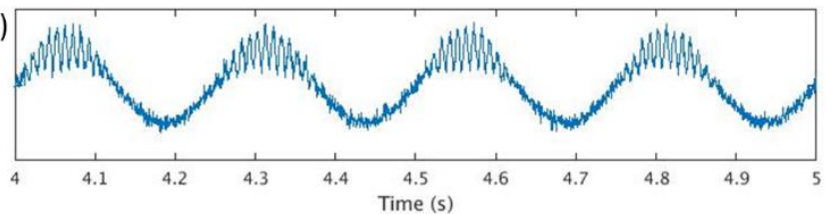

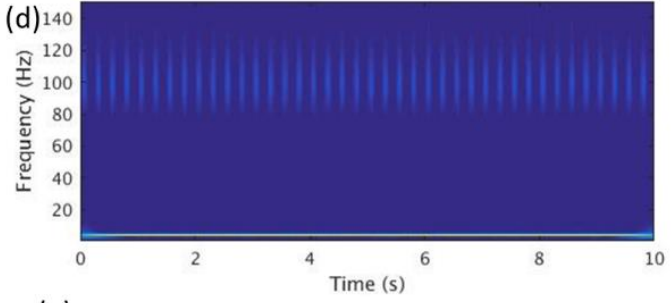

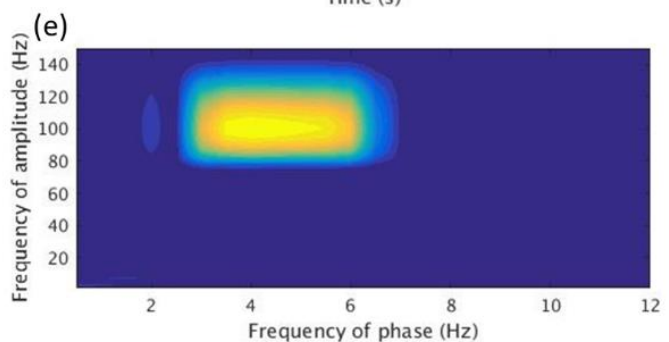

Fig. 3.3. Phase-amplitude CFC. a) A $4 \mathrm{~Hz}$ LFO rhythm. b) A $100 \mathrm{~Hz} \mathrm{HFO}$ rhythm. c) A composite signal with phase-amplitude CFC. The $100 \mathrm{~Hz}$ oscillations ride the peaks of the $4 \mathrm{~Hz}$ rhythm. d) A wavelet spectrogram of the composite signal. There is a $4 \mathrm{~Hz}$ band and $100 \mathrm{~Hz}$ modulated activity. e) An $\mathrm{I}_{\mathrm{cfc}}$ comodulogram computed using Tort's algorithm as described in the body of this section. There is an increase in the $\mathrm{I}_{\mathrm{cfc}}$ value in the region around $4 \mathrm{~Hz}$ in phase frequency and $100 \mathrm{~Hz}$ in amplitude frequency, as expected. 


\section{Chapter 4}

\section{Results}

\subsection{The Network Model Generates Recurrent SEDs}

Shown in Fig. 4.1 are the CRG membrane voltage outputs of each of the 16 cellular populations and the corresponding LFP. As can be seen from the LFP, with the parameter values provided in Tables 3.1 and 3.2, SEDs of various durations spontaneously arise. The SEDs often occur together in clusters, and are followed by a longer period of reduced activity characterized by slow uncoordinated low-amplitude oscillations.

During the SEDs, the membrane voltage of two or more of the pyramidal cells becomes visibly more ictal-like, as the clocks begin oscillating at a higher frequency. Two or more inhibitory interneurons also transition from a state of quiescence to bursting at high frequencies. This behavior is largely a manifestation of the clock-to-labile clock relationship between the pyramidal neurons and the interneurons.

The glial CRGs also behave differently in the SED and inter-SED periods. In the former, two or more of the microglia begin to oscillate more frequently. These then influence the oscillations of the astrocytes due to their clock-to-labile clock connectivity, causing astrocyte membrane voltage rises to appear clustered during SEDs.

In addition to evidence of intra-neuron and intra-glia interactions, we also observe signs of glianeuron connectivity. Particularly, the rises in astrocyte membrane voltage coincide well with increased pyramidal cell activity and interneuron bursting during SEDs. 


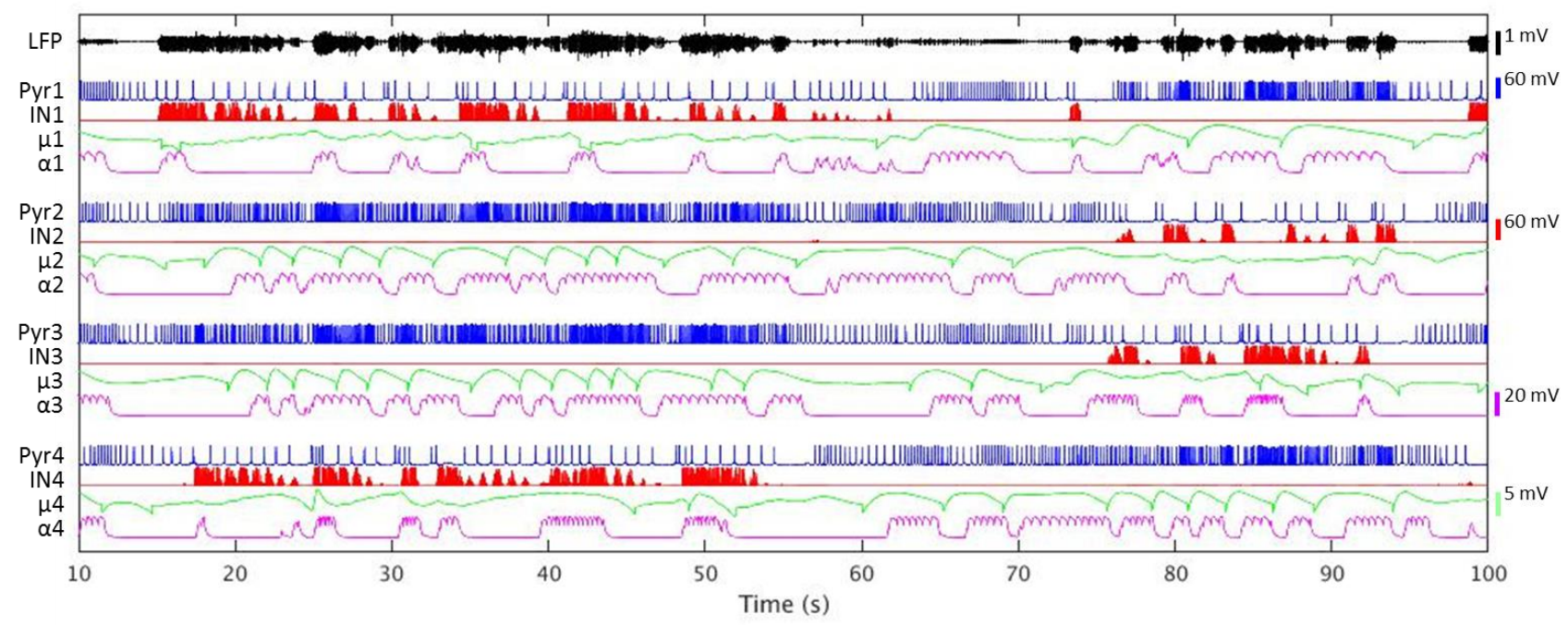

Fig. 4.1. Outputs of the network model under conditions of hyperexcitability. The top trace depicts the LFP, and the remaining lower traces are the membrane voltage $\left(\mathrm{V}_{\mathrm{m}}\right)$ outputs of each of the 16 CRGs, organized according to the subunits from which they originate. During the SEDs, two or more of the pyramidal populations exhibit increased activity, and two or more of the interneurons begin to spontaneously burst. Similarly, two or more microglia oscillate at higher frequencies, and two or more of the astrocytes exhibit clustered behavior. This collective activity gives rise to SEDs in the LFP.

\subsection{Different Processes Govern System Transitions in Short and Long Duration SEDs}

To gain a better understanding of the underlying distributions of the SEDs, the duration data was fit with a Gamma Mixture Model (GMM) containing two components (Fig. 4.2). SEDs with durations below $1.7 \mathrm{~s}$ had a greater overall probability of belonging to one distribution (distribution 2), and all other SEDs had a greater probability of belonging to the other (distribution 1). The distribution underlying the shorter durations decays to $5 \%$ of its peak value at $1.9 \mathrm{~s}$, thus validating the use of the 2 s biological threshold for the classification of SEDs as short or long [92] [127]. 


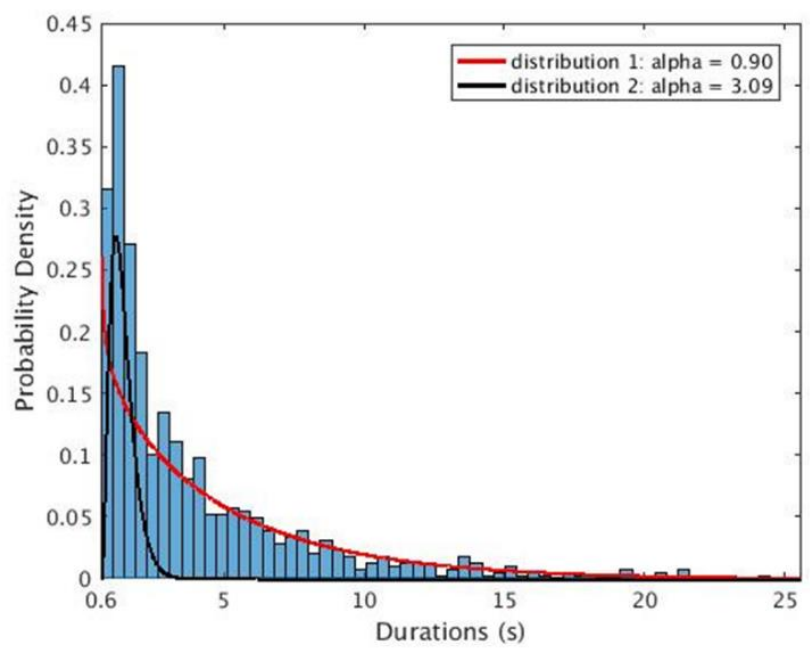

Fig. 4.2. Results of a Gaussian Mixture Model applied to SED duration data. Two components of a finite mixture model following gamma distributions were estimated using the EM algorithm. Shorter SEDs were found to predominantly belong to distribution 2, and longer SEDs were found to have a higher probability of belonging to distribution 1. Distribution 2 drops to 5\% of its peak value at an SED duration of $1.9 \mathrm{~s}$, thus validating the use of the biologically-observed $2 \mathrm{~s}$ threshold for separating short and long SEDs. The algorithm used for this implementation was obtained from [126].

To better understand the transition of the system into and out of the SEDs, the inter-SED and SED data were fitted with gamma distributions. The analysis was done separately for sSEDs and the region between them and 1 SEDs and the region between them. The histograms of the durations and the corresponding fits are shown in Fig. 4.3.

For sSEDs, the durations ranged from $0.201 \mathrm{~s}$ to $1.998 \mathrm{~s}$, and the distribution shape parameter, $\alpha$, of the gamma distribution was 7.34 (95\% CI: 6.47 8.33). This value is much greater than 1, indicating that the data follows a time-dependent deterministic process. The inter-sSED periods ranged from $0.200 \mathrm{~s}$ to $29.511 \mathrm{~s}$ in length, and the fit gave $\alpha=0.88$ (95\% CI: 0.751 .03 ). The 95\% confidence interval includes 1 , and the null hypothesis of a time-independent Poisson process cannot be rejected at the $5 \%$ significance level.

The 1SED and inter-1SED data showed a different result than the short duration data. The 1SED durations ranged from $2.006 \mathrm{~s}$ to $41.747 \mathrm{~s}$, and the shape parameter of the fitted distribution was $\alpha$ $=0.87$ (95\% 0.78 0.96), indicating random walk termination behavior. Finally, inter-1SED durations ranged from $0.110 \mathrm{~s}$ to $27.495 \mathrm{~s}$, and the shape parameter of the distribution was $\alpha=1.54$ 
(95\% 1.321 .79 ). In this case, the null hypothesis can again be rejected, and the data follows a deterministic process.
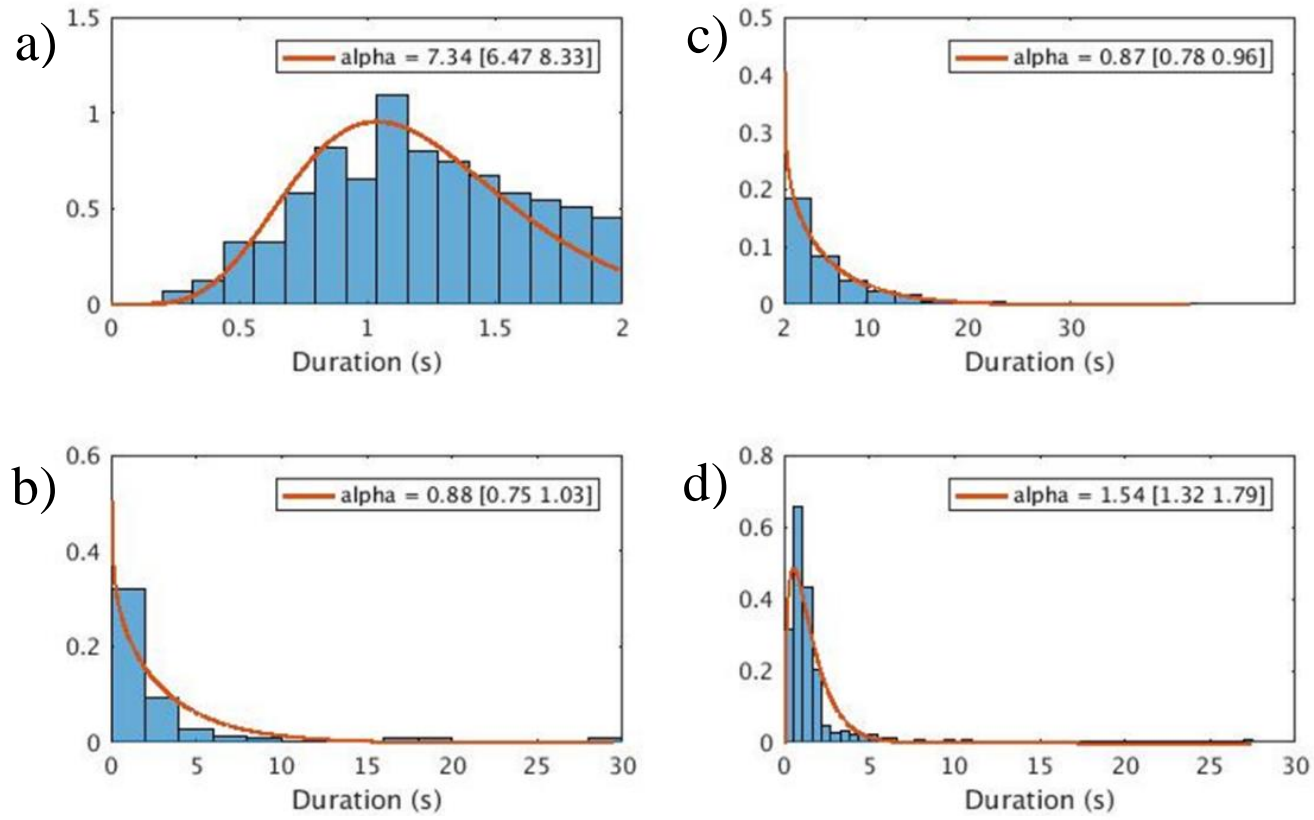

Fig. 4.3. Histograms and fitted gamma functions for SED and inter-SED duration data. State duration data was fit with gamma distributions and the resultant shape parameter, $\alpha$, was estimated. The estimate of $\alpha$ and the $95 \%$ confidence intervals (CIs) are presented in the subplot legends. a) sSEDs $(\mathrm{N}=457)$ : The shape parameter, $\alpha$, is 7.34, which indicates a deterministic termination process. b) inter-sSED $(\mathrm{N}=233)$ : The value of $\alpha$ lies within the $95 \%$ confidence interval of 1 , and thus indicates a Poisson-type process. $b$ ) 1SED $(\mathrm{N}=515)$ : The value of $\alpha$ is smaller than 1 , indicating random-walk type behavior. $\mathrm{d})$ inter-1SED $(\mathrm{N}$ $=279$ ): The value of $\alpha$ is 1.54 , indicating a deterministic process. SEDs and inter-SEDs show opposite dynamics for short and long duration discharges. sSEDs terminate in a deterministic manner whereas ISED terminate randomly. Conversely, inter-sSED states terminate randomly, whereas inter-lSEDs terminate deterministically. The fitting and estimation of $\alpha$ was computed using MATLAB's gamfit function.

\subsection{Glia-Neuron Interactions Play an Important Role in Controlling Hyperexcitability}

To better understand the effect that the glial populations have on hyperexcitability, the coupling from the glia to the neurons was varied and the corresponding LFP traces were plotted (Fig. 4.4). As seen in Fig. 4.4a, increasing the coupling coefficient between the astrocytes and the neurons 
results in an increasingly hyperexcitable system. When the coupling coefficient is zero, the astrocytes are disconnected from the neurons and the system is in a non-hyperexcitable state, displaying no SEDs. At low values of coupling, sSEDs and ISEDs begin to arise, with the latter dominating the LFP as the coupling becomes closer to its maximal value. At maximal coupling, the system is constantly in ISED states with very short inter-SED periods. This trend indicates that the presence of astrocytes is not only necessary for the occurrence of SEDs, but that they play an important role in controlling the duration of the discharge events.

Conversely, as the magnitude of the coupling coefficient between the microglia and the neurons is increased, the system becomes less hyperexcitable. In Fig. 4.4b, the LFPs are plotted as this connection is strengthened. When the microglia are disconnected from the neurons, the system is highly hyperexcitable and the LFP is dominated by ISEDs. As the connectivity reaches $70 \%$ of its maximal value, the LFP is composed of low-amplitude spiking, characteristic of inter-SED behavior. As the coupling is increased, the microglia are able to prune the synapses and have an inhibitory effect, thus preventing the occurrence of SEDs.

To see whether this microglial effect is the same under differing conditions, the excitability level of the system was increased and the effect on the LFP was observed with and without the microglial pruning. The increase in system excitability was achieved by increasing the excitatory connection (via coupling coefficient) from the astrocytes to the pyramidal populations. As can be seen in Fig. 4.5a, at low-to-moderate astrocyte-pyramidal cell coupling values, in the absence of pruning (blue LFP traces), SEDs arise spontaneously. The pruning (red LFP traces) reduces or even entirely removes these SEDs. At high astrocyte-pyramidal coupling values (Fig. 4.5b), the pruning actually results in longer discharges as compared to no pruning, eventually leading to continuous behavior similar to that seen in status epilepticus. At low-to-moderate hyperexcitability levels, the pruning decouples the neuronal populations and thus prevents SEDs from developing into more severe discharges. At higher levels however, this effect worsens the condition as it severs the negative feedback from the interneurons. This negative feedback is necessary for an SED to end, and as such, the system cannot leave the SED state. 


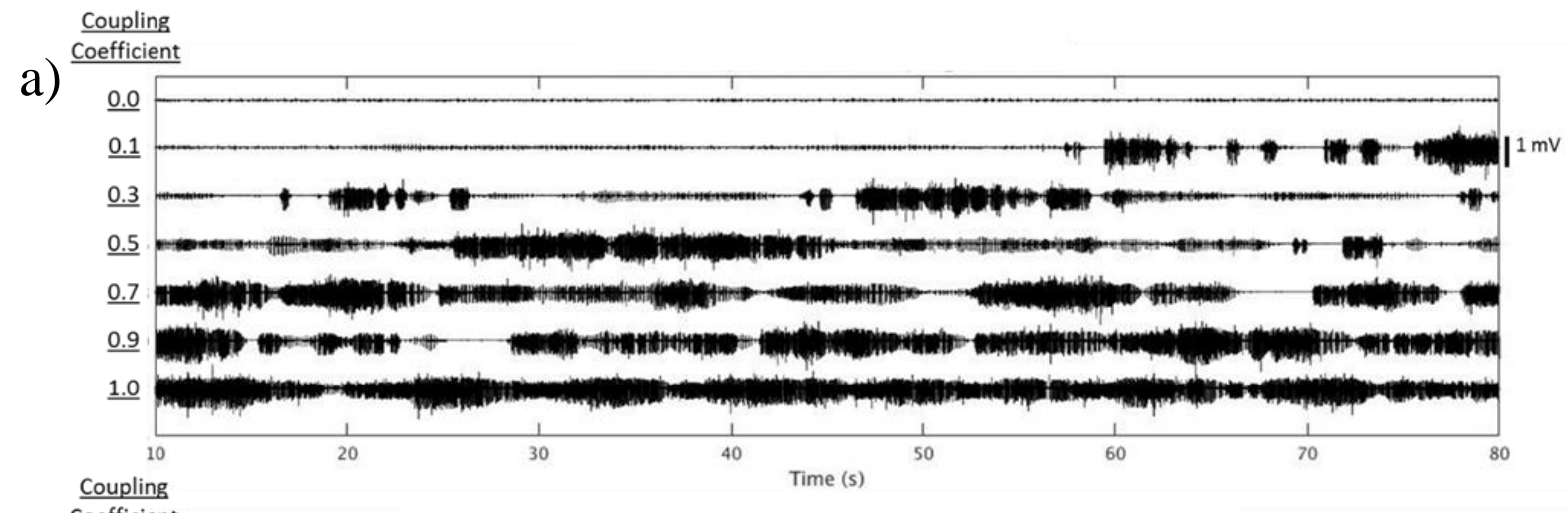

b)

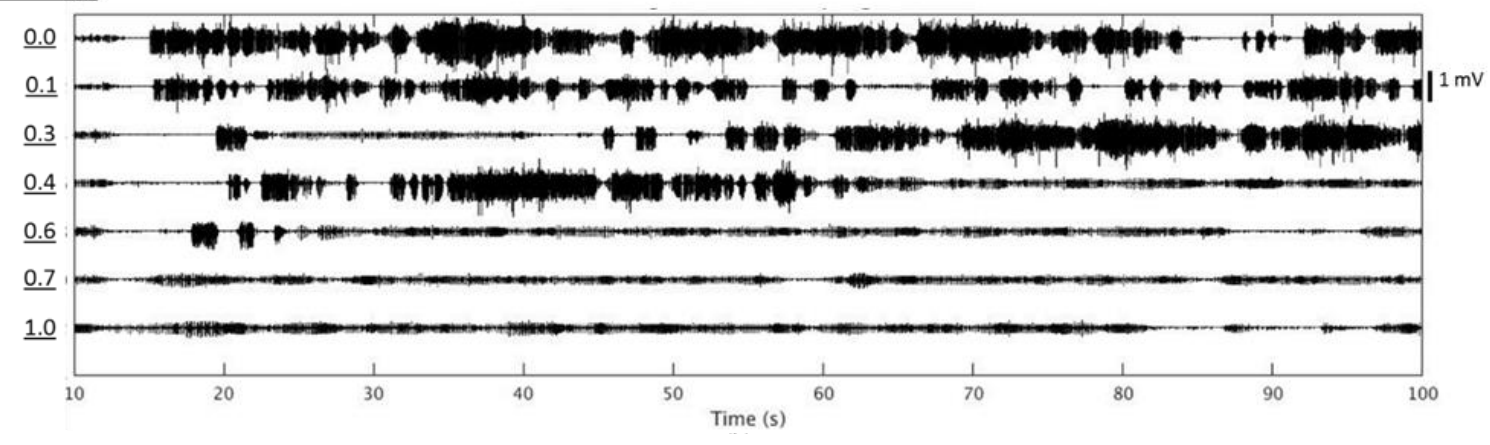

Fig. 4.4. Glial effects on network response as demonstrated from the LFP. a) Astrocytes-neurons coupling: With no connectivity between astrocytes and neurons (coupling coefficient $=0$ ), no SEDs are present in the LFP. As the coupling is strengthened, sSEDs and ISEDs begin to arise, with the latter becoming more prominent at higher coupling. As the coupling reaches its maximal value, the LFP shows continuous discharge activity as ISEDs become increasingly close to one another. b) Microglia-neurons coupling: Without microglial input to the neurons, the system is in a highly hyperexcitable state, characterized primarily by ISEDs. As the coupling is increased and the microglial pruning effects becomes more prominent, sSEDs begin to arise, while ISEDs begin to decrease in number. Upon reaching a coupling value of $70 \%$, the microglial inhibitory effect results in an LFP free of any SEDs. 
Coupling

Coefficient

a)

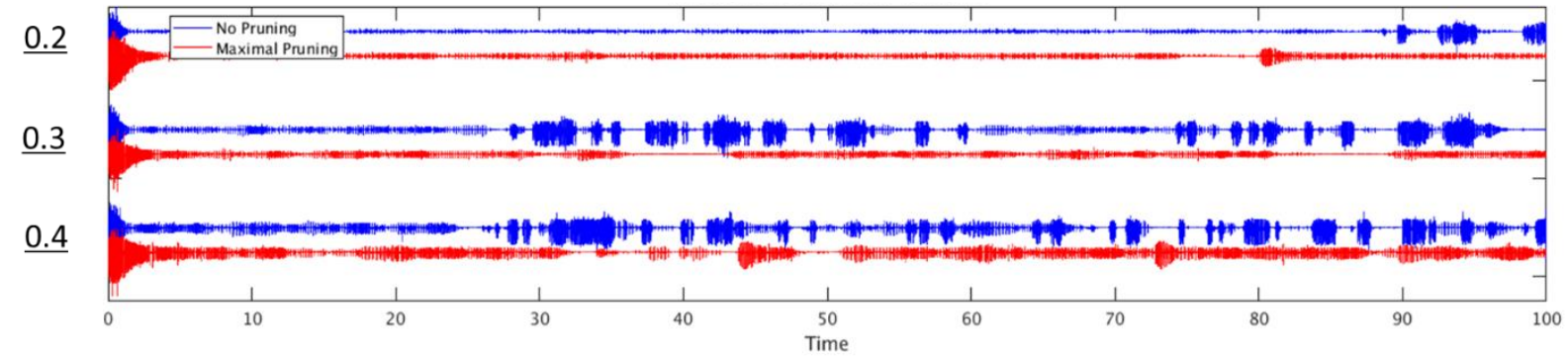

b) $\underline{0.8}$

$\underline{0.9}$

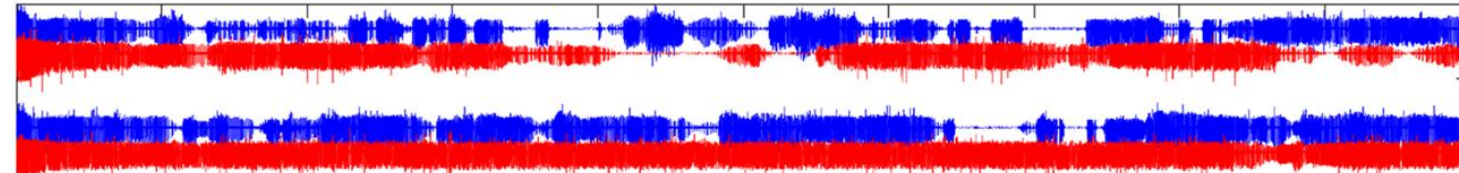

$\underline{1.0}$

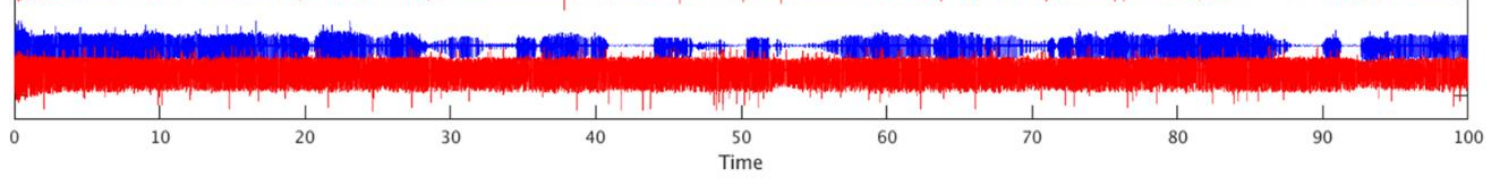

Fig. 4.5. Microglial pruning effect at various levels of system excitability as seen from the LFP. The excitability of the system was increased by increasing the coupling coefficient between the astrocytes and the pyramidal cell populations. For each coupling value, the simulations were ran in the absence of pruning (blue LFP traces) and in the presence of pruning (red LFP traces). a) At relatively low values of astrocytepyramidal cell coupling, in the absence of pruning, SEDs spontaneously arise. When pruning is present, these SEDs are either entirely abolished or reduced in frequency. b) At high values of coupling, in the absence of pruning, there are long SEDs with short inter-SED periods in between. With the pruning, the SEDs become longer and eventually become continuous at the maximal coupling coefficient value.

\subsection{Several Mechanisms Can Lead to Hyperexcitability}

To investigate other conditions leading to hyperexcitability, the connectivity between each of the different CRGs was varied, and the percent change in excitability, measured as the area under the excitability function, was computed.

The points corresponding to each type of connection were fitted with a line, not to establish a linear relationship, but to give an idea of the extent of the change in excitability as the coupling coefficient of the particular connection is varied. The slope $(m)$ and the Pearson's correlation coefficient $(r)$ obtained from these fits, as well as the variance $\left(\sigma^{2}\right)$ in excitability, for each of the connections are presented in Table 4.1. 
TABLE 4.1

ASSOCIATION OF CONNECTION TYPE WITH PERCENT CHANGE IN EXCITABILITY

\begin{tabular}{|c|c|c|c|}
\hline Connection & Slope $(\boldsymbol{m})$ & Pearson's Coefficient $(\boldsymbol{r})$ & Variance $\boldsymbol{\sigma}^{\mathbf{2}}\left(\mathbf{1 0}^{\mathbf{3}}\right)$ \\
\hline astro-neuron & 3.51 & 0.65 & 26.09 \\
\hline pyramidal-astro & 0.70 & 0.77 & 0.75 \\
\hline pyramidal-micro & -0.56 & 0.64 & 0.71 \\
\hline pyramidal-interneuron & 5.79 & 0.96 & 32.80 \\
\hline micro-astro & 3.95 & 0.80 & 18.87 \\
\hline astro-micro & 1.11 & 0.78 & 1.88 \\
\hline interneuron-astro & 0.00 & 0.00 & 0.50 \\
\hline interneuron-micro & 3.50 & 0.89 & 12.52 \\
\hline interneuron-pyramidal & -0.62 & 0.75 & 0.73 \\
\hline micro-neuron & -0.82 & 0.87 & 0.77 \\
\hline micro-micro & 0.58 & 0.20 & 7.75 \\
\hline astro-astro & 3.22 & 0.93 & 11.08 \\
\hline
\end{tabular}

\subsubsection{Excitatory Connections}

In most cases, strengthening the excitatory connections has the effect of increasing the hyperexcitability of the system (Fig. 4.6a). The excitatory connection with the greatest impact $(m$ $=5.79)$ and strongest correlation $(r=0.96)$ is that from the pyramidal cells to the interneurons. The pyramidal-interneuron connection is essential to the generation of SEDs, because of the clockto-labile clock relationship between the two cell types. Severing the connection by setting the coupling to zero, results in complete silence of the labile inhibitory interneurons, which require an input to undergo bursting. Without any interneuron activity, the system is not able to transition out of the low-excitability state.

The two other connections with large slope and variance values both involve the astrocyte CRG populations. The excitatory connection between the microglia and the astrocytes has a great impact on the excitability $(m=3.95)$. This can be attributed at least in part to the clock-to-labile clock relationship that exists between the two populations. At low coupling values, the input to the astrocytes is below the threshold required for activation of the labile clock and there is thus little astrocyte activity. Under these conditions when the astrocytes are not oscillating, SEDs do not arise and the system remains in a non-hyperexcitable state. As discussed in the previous section, the connection between the astrocytes and the neurons has a strong effect on system hyperexcitability $(m=3.50)$. 
Strengthening the connection from the pyramidal cells to the microglia has the opposite effect, resulting in a reduction in system excitability $(m=-0.56)$. This effect may be due to the pruning functionality of the microglia, which becomes more prominent as they become more excited by input from the pyramidal neurons.

\subsubsection{Inhibitory Connections}

In two of the four inhibitory connections, increasing the magnitude of the coupling coefficient resulted in a decrease in excitability (Fig. 4.6b). The first of these connections was from the microglia to the neurons $(m=-0.82)$, and the other was the connection from the interneurons to pyramidal cells $(m=-0.62)$.

Strengthening the connection from the inhibitory interneurons to the microglia, on the other hand, results in an increase in excitability $(m=3.50)$. This may be due to a microglial disinhibition effect, where the microglia are inhibited. As such, the pruning becomes less effective and the system becomes increasingly hyperexcitable.

\subsubsection{Gap Junctions}

Strengthening the coupling values associated with the gap junctions resulted in an increase in excitability for both glial populations (Fig. 4.6c). In the case of the astrocytes, the increase in excitability was evident from the slope $(m=3.22)$, the Pearson's correlation coefficient $(r=0.93)$, and the variance $\left(\sigma^{2}=11.08 \times 10^{3}\right)$. This increase in excitability can be attributed to the excitatory role of the astrocytes in the network. As the gap junctional connections become stronger, the astrocytes become more excited, which in turn excites other populations. As discussed above, the astrocyte-neuron connection, for example, is strongly associated with hyperexcitability.

While the micro-micro connection has small slope $(m=0.58)$ and correlation values $(r=0.20)$, the variance is the $5^{\text {th }}$ largest of all connections $\left(\sigma^{2}=7.75 \times 10^{3}\right)$. This is due to an irregular trend, with a peak occurring when the percent change in coupling is around $25 \%$, followed by a drop and plateau in excitability. This phenomenon can be attributed to the mixed excitatory and inhibitory connections that the microglia have with other populations. As the coupling is first increased, through its excitatory connections, the energy change of the astrocytic voltage signals increases, resulting in increased system hyperexcitability. As the coupling begins to exceed $25 \%$, the direct connection between the microglia causes the microglial signal energy change to be 
larger than that of the astrocytes. As such, the inhibitory behavior of the microglia becomes more prominent and results in a drop in the excitability. These two antagonistic effects balance as the overall excitability of the system plateaus at a $100 \%$ increase. 

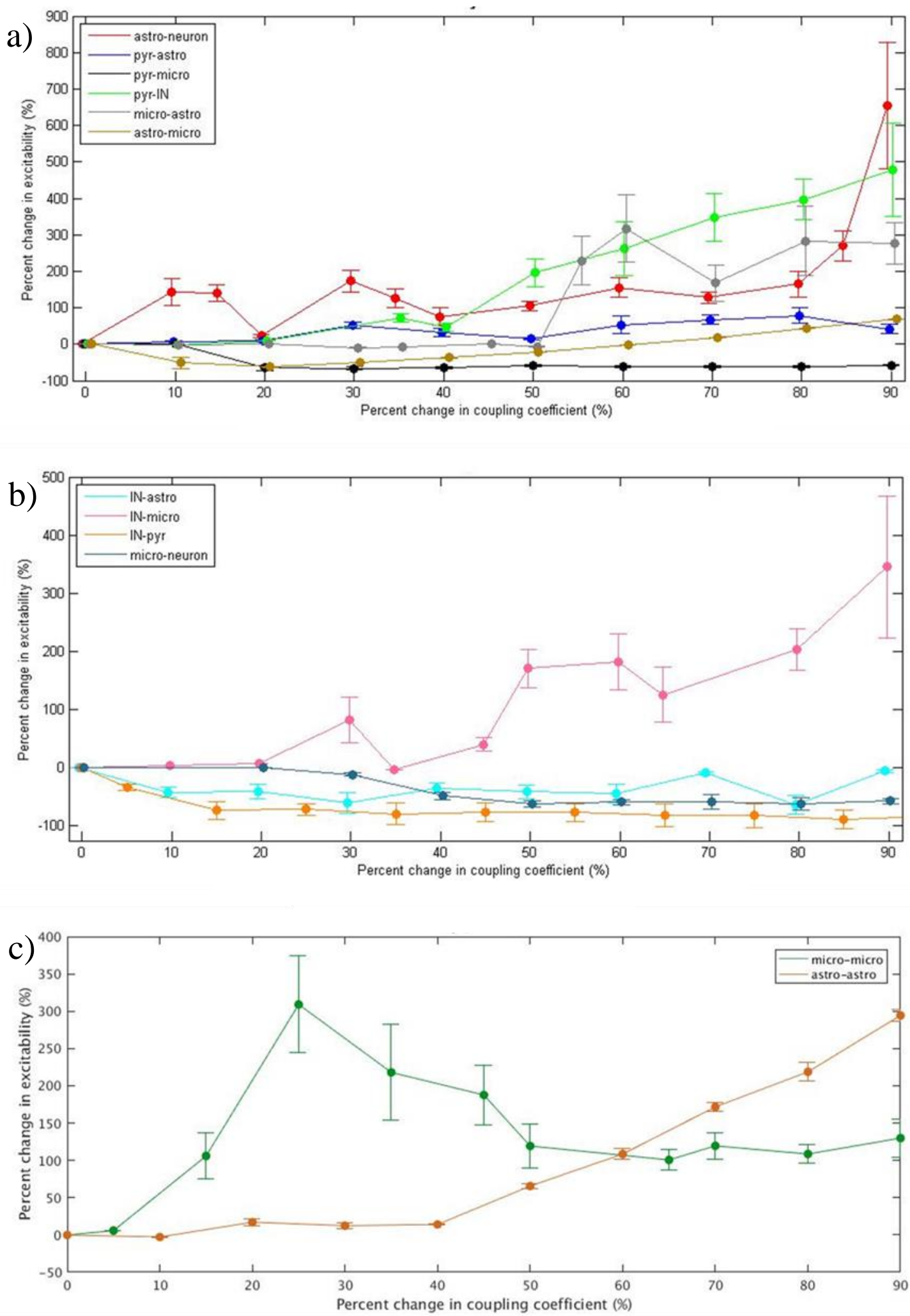

Fig. 4.6. The effects of connectivity on network excitability. The percent change in excitability is computed as the coupling coefficient is varied between a pair of populations. a) Excitatory connections: the 
three excitatory connections most strongly associated with excitability are those from the pyramidal cells to interneurons, from the astrocytes to the neurons, and from the microglia to the astrocytes. While strengthening the coupling of those connections results in an increase in excitability, strengthening the connection from pyramidal cells to interneurons results in a reduction in excitability. b) Inhibitory connections: the connections from interneurons to pyramidal cells and from the microglia to the neurons are associated with a decrease in system excitability, whereas the interneuron inhibition of the microglia results in an increase in the excitability. c) Gap junctions: Increasing the coupling associated with intraastrocyte and intra-microglia gap junctions result in an overall increase in the system excitability. Because the microglia have excitatory and inhibitory roles, the excitability of the microglial gap junctions connection peaks at coupling values near $25 \%$ before settling at a lower excitability level. All corresponding association values (slope, Pearson's correlation coefficient, and variance) can be found in Table 4.1. Error bars in the plots represent the standard error of the mean (SEM).

\subsection{SEDs Exhibit Delta-HFO and sSEDs Exhibit Theta-HFO Phase- Amplitude Coupling}

To study the cross-frequency interactions in the model, the $I_{\text {cfc }}$ on both sSEDs and ISEDs was computed. The $\mathrm{I}_{\mathrm{cfc}}$ is a measure of phase-amplitude coupling, quantifying the extent to which the phase of a lower frequency modulates the amplitude of a higher frequency.

In the case of the 1SED, phase-amplitude coupling is dominant between the delta frequency range and the ripple and fast ripple ranges, in particular between 3-4 Hz and 150-350 Hz (Fig. 4.7b). In addition, although weaker than the delta-HFO modulation, there is also some theta-HFO coupling, particularly between the 6-7 Hz range and the fast ripple range. In comparison, the dominant phase-amplitude coupling in the sSED is in the form of theta-HFO modulation (Fig. 4.7a). 

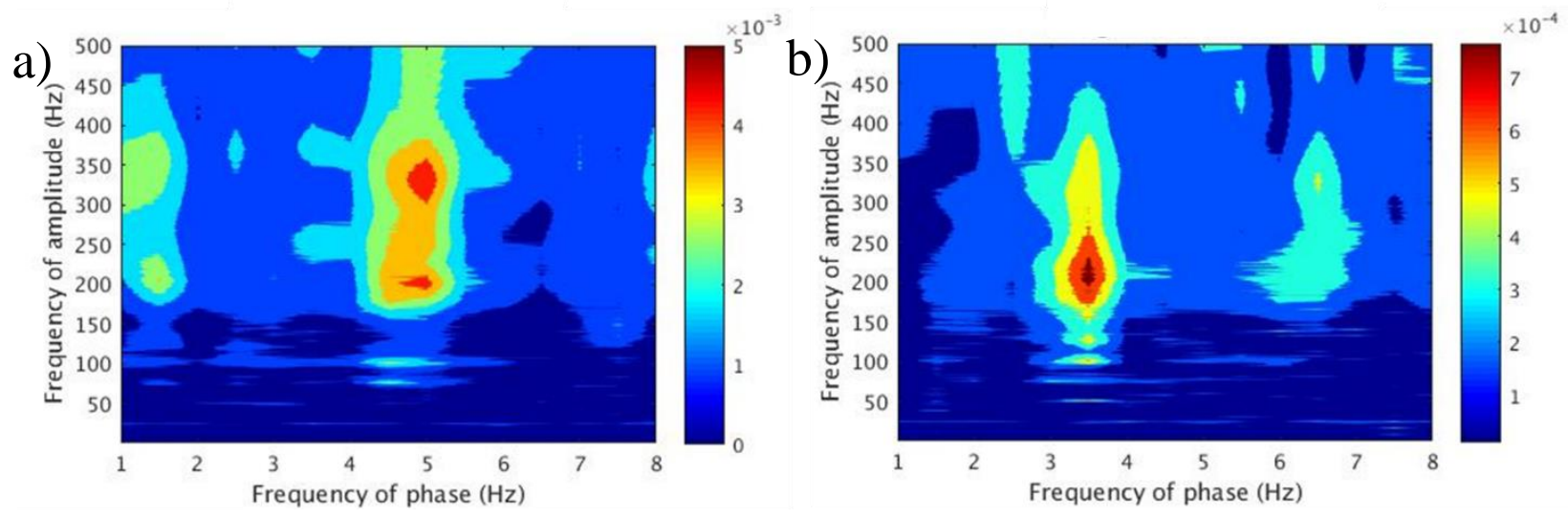

Fig. 4.7. Cross Frequency Coupling Index $\left(I_{\text {cfc }}\right)$ of SEDs. a) The $I_{\text {cfc }}$, computed for an sSED, shows evidence of theta-HFO phase-amplitude coupling. b) The $\mathrm{I}_{\mathrm{cfc}}$, computed for an 1SED, shows evidence of delta-HFO phase-amplitude coupling. 


\section{Chapter 5}

\section{Discussion}

\subsection{General}

Using a meso-level modeling approach, we developed a neuroglial hippocampal network capable of producing SEDs of similar characteristics to those observed in many experimental models of epilepsy. The occurrence of an SED is accompanied by observable changes in the activities of both neuronal and glial populations. The pyramidal cells and the interneurons exhibit bursting at a higher frequency than the baseline, as is seen in experimental SLEs [119]. Similarly, the astrocytes display oscillations at higher frequencies and larger amplitude [51] [52].

The model generated SEDs between $0.201 \mathrm{~s}$ and $41.747 \mathrm{~s}$ in duration, and even displayed continuous discharging under certain parameterizations. This range is larger than that observed in the original SED model, where SEDs were between $2 \mathrm{~s}$ and $8 \mathrm{~s}$ [119]. Zalay et al. attributed that shortcoming to a small network size. They argued that just as hippocampal slices generate SEDs of much shorter durations (seconds) than the intact hippocampus (minutes), a larger network size can sustain longer discharges. Indeed this hypothesis appears to be correct, as the number of CRGs went from 4 in the original model to 16 in this one.

\subsection{Glial Cells Play an Important Role in Hyperexcitability}

This neuroglial model has demonstrated many different avenues to hyperexcitability, and provides insight into the lesser-understood role of astrocytes and microglia in epilepsy.

The astrocyte populations, in the model, play a key role in the hyperexcitability of the system. As the coupling between the astrocytes and the neurons was increased, the system became more hyperexcitable and eventually entered a state characterized by little time between long SEDs, such that the LFP appears to consist of a continuous discharge (Fig. 4.4a). This behavior is consistent with the astrocytic release of substances, such as glutamate, aspartate, and D-serine, known to 
cause synchronous neuronal depolarizations and epileptic behavior [62] [59] [54]. The gap junctions of the astrocytes were also found to affect the hyperexcitability of the system. This has been demonstrated in studies of cerebral tissue from animal seizure models and human patients, where there was an up-regulation of glial gap junctional mRNA and protein [128].

The microglial populations were found to modulate the hyperexcitability of the system. As the coupling between the microglia and the neurons was strengthened, there were fewer SEDs and they were of shorter duration. Eventually, when the coupling between the microglia and the neurons was sufficiently high, all of them were abolished. This is consistent with the neuroprotective role of microglia in regulating synaptic activity. Microglia are known to make contact with hyperactive neurons to reduce EPSC frequency and to downregulate their activity [129] [130]. Furthermore, blocking certain functions of microglia have been shown to be related to the occurrence of seizures. Eyo et al., for example, found that blocking a microglial receptor (P2Y12) essential for the outgrowth of microglial processes results in a worsened seizure phenotype [81]. In another study, Derecki et al. found that blocking microglial phagocytosis promotes the development of Rett Syndrome, a condition that most often results in seizures [82]. Interestingly in Mecp2-null mice (a model of Rett Syndrome) and other pathological conditions, there is an up-regulation of certain microglial gap junction proteins [78] [131]. The microglial gap junctions in the model similarly have a role in elevating the excitability level of the system.

The connection between the microglial and astrocytic populations in the model was found to be one of the most essential for the development of hyperexcitable conditions. At low connectivity levels, the system does not generate any visible SEDs in the LFP. This is not entirely surprising as it has been found that under pathological conditions, microglia can release ATP that binds on astrocytes and causes an increase in excitatory transmission via a metabotropic glutamate receptordependent mechanism [73].

Whether microglia are helpful or harmful in pathological conditions remains unclear, and both neuroprotective and neurodegenerative roles in epilepsy have been identified [66] [69]. This was also observed in the model, as the effect of the microglial pruning mechanism was not the same under different conditions. At lower excitability levels, under weak coupling between the astrocyte and pyramidal cell populations, the presence of pruning acted to abolish or attenuate SEDs by decoupling neuronal populations. At higher excitability levels, however, the presence of pruning 
actually led to longer (or even continuous) discharges. This occurred due to the severing of the inhibitory feedback that would be necessary for ending a seizure. Such a mechanism may be a candidate for the physiological occurrence of long or continuous seizures.

While the model sheds some light into the important role of glial populations in epileptic conditions, it certainly does not negate the significance of neuronal populations. In fact, according to the model, the bidirectional connections between the pyramidal cell and interneuron populations are arguably the most strongly associated with the excitability level of the system (Fig. 4.6a and 4.6b). This is consistent with the classical view of epilepsy as being caused by a disruption in the balance between excitation and inhibition in neurons [132] [133].

\subsection{Comparison of Short and Long SEDs}

The neuroglial model generates SEDs of various durations, with longer SEDs being more prevalent in more hyperexcitable conditions. A Gamma Mixture Model with two components revealed that there may in fact be two underlying distributions to the model-generated SEDs, one primarily representing shorter discharges and another longer ones. Interestingly, the distribution underlying sSEDs drops to $5 \%$ of its peak value at a duration of $1.9 \mathrm{~s}$, which is close to a physiologicallydetermined separation threshold of 2 s [127] [92].

Applying distributions individually to the short and long duration discharge data reveals that they exhibit opposing state dynamics. SSEDs have $\alpha>1$ and inter-sSEDs have $\alpha=1$, while 1SEDs have $\alpha<1$ and inter-1SEDs have $\alpha>1$. This result has two important implications. First, it suggests that the longer the system remains in an SSED, the more likely that it will transition into an SED-free epoch, whereas the longer that it remains in an ISED, the more likely that it will remain in that state. Furthermore, the inter-1SED state is more likely to terminate the longer the system remains in the state. This implies that once the system enters an ISED, it is likely that it will remain at a high excitability level characterized by long discharges with short breaks in between. As such, it is more harmful to enter or to be in ISED states as compared to sSEDs. Second, because intersSEDs follow a Poisson-type process, it may not be possible to predict when a short discharge will occur. Inter-1SEDs however terminate in a time-dependent fashion due to deterministic 
mechanisms that may be predictable. Collectively these results suggest that 1SEDs are more pathological, but luckily it may be possible to predict them.

These differing underlying dynamics are further supported by the different $\mathrm{I}_{\mathrm{cfc}}$ results of the short and long duration discharges. While both types of SEDs show some delta-HFO and theta-HFO phase-amplitude coupling, the dominant form is delta-HFO in the case of ISEDs and theta-HFO in the case of sSEDs. Based on the gamma distribution results, this implies that delta-HFO crossfrequency interactions underlie pathological behavior. Interestingly, this is precisely what was found in Mecp2-deficient mice, where rescuing the animal by reintroduction of the Mecp2 gene resulted in a reduction of delta-HFO interactions [92]. Furthermore, the presence of delta-HFO coupling has been identified as a marker for epileptogenesis in humans [33].

\subsection{Limitations}

Below are three limitations of the neuroglial model in its current form. These are briefly addressed in Chapter 6 while discussing future work.

- Modeling scale: All three stages of the CRG are higher-level abstractions of the actual underlying physiological phenomena. While the PDMs capture the functional properties, such as the coding and transformation operations of cellular populations, they provide no structural information about the modeled system. The second stage, the ring device, is a lumped modeling approach, that while is well-suited for modeling the endogenous rhythms of cellular populations, does not concern itself with the underlying structural details of the system. Third, the mapper is a look-up table that can generate realistic outputs, but it too contains no information about the underlying structure or action of the cell membrane. As such, the neuroglial model can elucidate phenomena, but can give little information about the molecular causes. For example, we found that the connection between the astrocytes and the neurons is important in hyperexcitability, but we cannot say whether the effect is mediated by dysfunctional $\mathrm{K}^{+}$buffering or due to irregular glutamate signaling. Such details can be better understood through the use of microlevel models. 
- No long-term plasticity: This neuroglial model is limited by the lack of an explicit form of long-term plasticity. Influenced by input from the neuronal populations, the microglial pruning mechanism that is currently implemented can reversibly weaken synapses. However, it is limited in that, first, it is unable to strengthen synapses, and second, it cannot maintain its effect for much longer than a few seconds.

- The omission of higher-order PDMs: While the use of an integrating mode is justified because it was found to have the greatest contribution to the hippocampal response and because of the integrative role of glial cells, the other modes may still play an important role in neuroglial systems [52] [121]. They can have various differentiating characteristics, and in combination, they have been shown to code for position, velocity, and acceleration in various physiological systems [134] [135]. 


\section{Chapter 6}

\section{Conclusion}

\subsection{Summary and Significance}

Presented in this thesis is a mesoscale coupled oscillators neuroglial model capable of generating SEDs with similar characteristics to those previously observed in SLEs and in human epileptic seizures. The model sheds light on the importance of astrocytic and microglial populations as contributors to hyperexcitability and to the generation of short and long duration SEDs. Analysis reveals differences in phase-amplitude cross-frequency coupling characteristics and in the underlying distributions between short and long SEDs, suggesting a more pathological role for the latter.

The significance of this work is as follows:

1. It highlights the importance of targeting glial populations in the development of AEDs and other treatment strategies.

2. It provides a suitable platform for testing different treatment strategies, owing to the model's ability to closely reproduce the properties, underlying mechanisms, and dynamics of epileptic behaviour.

3. It provides an appropriately (meso-)scaled model that facilitates implementation in hardware for use in neuromodulation strategies and neuroprosthetics in the treatment of epilepsy and other neurological diseases.

\subsection{Future Directions}

In consideration of the current limitations of the model and the desire for future clinical relevance, below are some possible areas of future work:

- A microscale-mescoscale hybrid modeling approach: While the strength of the CRG is in its level of abstraction, this becomes a drawback if knowledge of molecular processes or mechanisms is desired. This neuroglial model has elucidated certain high- 
level phenomena, such as the contribution of astrocytes to neuronal hyperexcitability. Combining this model with conductance-based compartmental models may provide insight into the specifics of this mechanism. A hybrid model that utilises microglial CRGs is currently being developed by lab colleague Vasily Grigorovsky.

- Introduction of long-term plasticity: The phenomena of long term potentiation (LTP) and long term depression (LTD) can be introduced as a set of dynamical equations to be solved along with the current model equations. The implementation can take many forms, such as a simpler two-threshold /ABS model or more complex spike timing dependant plasticity (STDP) models [136] [137].

- The addition of higher order PDMs: As described in Chapter 3, the CRGs in the neuroglial model contained only an integrating mode. Including the higher-order modes can be achieved by solving a system of integro-differential equations.

- Machine Learning Algorithms: Artificial Neural Networks (ANNs) and other machine learning algorithms draw inspiration from biological systems. Some work has been done to add glial cells to these neural networks [138]. A possible avenue for investigation is the incorporation of this neuroglial model, as a dynamical system, at the core of Deep Neural Networks (DNNs). To create the CRG, non-parametric modeling (in the form of kernels) was combined with parametric modeling (in the form of an oscillator ring device). This presents another opportunity to reap the benefits of other non-parametric models, such as ANNs.

- Testing neuromodulation strategies: Zalay et al. and Colic et al. have both created signals that were able to reduce the severity of SEDs when tested in the original SED model [101] [102]. This expanded model, which now incorporates glial populations and HFOs, may present a more biologically-relevant platform for the testing of neuromodulation strategies.

- Hardware implementation: This model generates biomimetic signals spanning a wide range of frequency bands. These signals may prove to be effective for neuromodulation purposes in experimental systems. While these signals can be stored in memory and used for stimulation, more effective systems will incorporate real-time feedback. This can be achieved by implementing the model in hardware (ex. FPGAs) for improved portability and increased computational efficiency. 
- Experimental validation: Computational models gain strength by generating hypotheses that prove to have some merit in experimental systems. Alternatively, if the hypotheses are disproved, then the model will benefit from making modifications that account for these results. This neuroglial model has led to various testable hypotheses, particularly related to the role of glial cells in hyperexcitability. These can be tested given the wide range of experimental techniques and resources available to us through our research partners. 


\section{References}

[1] F. Lopes da Silva, W. Blanes, S. Kalitzin, J. Parra, P. Suffczynski and D. Velis, "Epilepsies as dynamical diseases of brain systems: basic models of the transition between normal and epileptic activity," Epilepsia, vol. 44, no. 12, pp. 72-83, 2003.

[2] F. Jensen, "Epilepsy as a spectrum disorder: Implications from novel clinical and basic neuroscience," Epilepsia, vol. 52, no. 1, pp. 1-6, 2011.

[3] R. Fisher, C. Acevedo, A. Arzimanoglou, A. Bogacz, J. Cross, C. Elger, J. Engel Jr, L. Forsgren, J. French, M. Glynn, D. Hesdorffer, B. Lee, G. Mathern, S. Moshé, E. Perucca, I. Scheffer, T. Tomson, M. Watanabe and S. Wiebe, "ILAE official report: a practical clinical definition of epilepsy," Epilepsia, vol. 55, no. 4, pp. 475-482, 2014.

[4] A. Ngugi, C. Bottomley, I. Kleinschmidt, J. Sander and C. Newton, "Estimation of the burden of active and life-time epilepsy: a meta-analytic approach," Epilepsia, vol. 51, no. 5, pp. 883-890, 2010 .

[5] S. Shorvon, "Epidemiology, classification, natural history, and genetics of epilepsy," The lancet, vol. 336, no. 8707, pp. 93-96, 1990.

[6] M. England, C. Liverman, A. Schultz and L. Strawbridge, Epilepsy across the spectrum, Washington: National Academies Press, 2012.

[7] R. Fisher, J. Cross, J. French, N. Higurashi, E. Hirsch, F. Jansen, L. Lagae, S. Moshé, J. Peltola, E. Roulet Perez, I. Scheffer and S. Zuberi, "Operational classification of seizure types by the International League Against Epilepsy: Position Paper of the ILAE Commission for Classification and Terminology," Epilepsia, vol. 58, no. 4, pp. 522-530, 2017.

[8] C. Skidmore, "Adult focal epilepsies," Contiuum: Lifelong learning in neurology, vol. 22, no. 1, pp. 94-115, 2016.

[9] G. Cascino and J. Sirven, Adult epilepsy, Wiley-Blackwell, 2011.

[10] R. Hrachovy and J. Frost Jr, "The EEG in selected generalized seizures," Journal of clinical neurophysiology, vol. 23, no. 4, pp. 312-332, 2006.

[11] E. Trinka, H. Cock, D. Hesdorffer, A. Rossetti, I. Scheffer, S. Shinnar, S. Shorvon and D. Lowenstein, "A definition and classification of status epilepticus--Report of the ILAE Task Force on Classification of Status Epilepticus," Epilepsia, vol. 56, no. 10, pp. 1515-1523, 2015.

[12] P. Smith, "Introduction: The causes of epilepsy," Epilepsia, vol. 53, no. s4, pp. 1-2, 2012.

[13] C. Marini, I. Scheffer, R. Nabbout, A. Suls, P. De Jonghe, F. Zara and R. Guerrini, "The genetics of Dravet syndrome," Epilepsia, vol. 52, no. s2, pp. 24-29, 2011. 
[14] J. Christensen, "Traumatic brain injury: risks of epilepsy and implications for medicolegal assessment," Epilepsia, vol. 53, no. 4, pp. 43-47, 2012.

[15] B. Michael and T. Solomon, "Seizures and encephalitis: clinical features, management, and potential pathophysiologic mechanisms," Epilepsia, vol. 53, no. 4, pp. 63-71, 2012.

[16] N. Villeneuve, "Quality-of-life scales for patients with drug-resistant partial epilepsy," Revue Neurologique, vol. 160, no. 1, pp. 376-393, 2004.

[17] A. Wagner, K. Bungay, M. Kosinski, E. Bromfield and B. Ehrenberg, "The health status of adults with epilepsy compared with that of people without chronic conditions," Pharmacotherapy, vol. 16, no. 1, pp. 1-9, 1996.

[18] A. Jacoby and J. Austin, "Social stigma for adults and children with epilepsy," Epilepsia, vol. 48, no. 9, pp. 6-9, 2007.

[19] V. Novak, "Epilepsy treatment introduction," Clinical Therapeutics, vol. 39, no. 8, pp. 105-106, 2017.

[20] S. Noachtar and I. Borggraefe, "Epilepsy surgery: a critical review.," Epilepsy and behavior, vol. 15, no. 1, pp. 66-72, 2009.

[21] J. Téllez-Zenteno, R. Dhar and S. Wiebe, "Long-term seizure outcomes following epilepsy surgery: a systematic review and meta-analysis," Brain, vol. 128, no. 5, pp. 1188-1198, 2005.

[22] A. Mansouri, A. Fallah and T. Valiante, "Determining surgical candidacy in temporal lobe epilepsy," Epilepsy research and treatment, 2012.

[23] J. Janszky, I. Janszky, R. Schulz, M. Hoppe, F. Behne, H. Pannek and A. Ebner, "Temporal lobe epilepsy with hippocampal sclerosis: predictors for long-term surgical outcome," Brain, vol. 128, no. 2, pp. 395-404, 2005.

[24] E. Behrens, J. Schramm, J. Zentner and R. König, "Surgical and neurological complications in a series of 708 epilepsy surgery procedures," Neurosurgery, vol. 41, no. 1, pp. 1-9, 1997.

[25] R. Surges and C. Elger, "Reoperation after failed resective epilepsy surgery," Seizure, vol. 22, no. 7, pp. 493-501, 2013.

[26] R. Elliott, A. Morsi, O. Tanweer, B. Grobelny, E. Geller, C. Carlson, O. Devinsky and W. Doyle, "Efficacy of vagus nerve stimulation over time: review of 65 consecutive patients with treatmentresistant epilepsy treated with VNS > 10 years," Epilepsy and behavior, vol. 20, no. 3, pp. 478483, 2011.

[27] R. Fisher, V. Salanova, T. Witt and R. Worth, "Electrical stimulation of the anterior nucleus of thalamus for treatment of refractory epilepsy," Epilepsia, vol. 51, no. 5, pp. 899-908, 2010. 
[28] E. Bae, L. Schrader, K. Machii, M. Alonso-Alonso, J. Riviello Jr, A. Pascual-Leone and A. Rotenberg, "Safety and tolerability of repetitive transcranial magnetic stimulation in patients with epilepsy: a review of the literature," Epilepsy and behavior, vol. 10, no. 4, pp. 521-528, 2007.

[29] W. Theodore and R. Fisher, "Brain stimulation for epilepsy," in Operative neuromodulation, New York, Springer, 2007, pp. 261-272.

[30] D. Durand and M. Bikson, "Suppression and control of epileptiform activity by electrical stimulation: a review," Proceedings of the IEEE, vol. 89, no. 7, p. 2001, 1065-1082.

[31] B. Leuner and E. Gould, "Structural plasticity and hippocampal function," Annual review of psychology, vol. 61, pp. 111-140, 2010.

[32] D. Kullmann, "Interneuron networks in the hippocampus," Current opinion in neurobiology, vol. 21, no. 5, pp. 709-716, 2011.

[33] M. Guirgis, Y. Chinvarun, M. Del Campo, P. Carlen and B. Bardakjian, "Defining regions of interest using cross-frequency coupling in extratemporal lobe epilepsy patients," Journal of neural engineering, vol. 12, no. 2, 2015.

[34] G. Buzsáki, Rhythms of the brain, New York: Oxford University Press, 2006.

[35] M. Avoli, "The epileptic hippocampus revisited: Back to the future," Epilepsy currents, vol. 7, no. 4, pp. 116-118, 2007.

[36] B. McNaughton, C. Barnes and J. O'Keefe, "The contributions of position, direction, and velocity to single unit activity in the hippocampus of freely-moving rats," Experimental brain research, vol. 52, no. 1, pp. 41-49, 1983.

[37] P. Hemond, D. Epstein, A. Boley, M. Migliore, G. Ascoli and D. Jaffe, "Distinct classes of pyramidal cells exhibit mutually exclusive firing patterns in hippocampal area CA3b," Hippocampus, vol. 18, no. 4, pp. 411-424, 2008.

[38] C. Le Duigou, J. Simonnet, M. Telenczuk, D. Fricker and R. Miles, "Recurrent synapses and circuits in the CA3 region of the hippocampus: an associative network," Frontiers in cellular neuroscience, vol. 7, no. 262, 2013.

[39] T. Freund and G. Buzsaki, "Interneurons of the hippocampus," Hippocampus, vol. 6, pp. 347-470, 1996.

[40] A. Sik, M. Penttonen, A. Ylinen and G. Buzsaki, "Hippocampal CA1 interneurons: An in vivo intracellular labeling study," The journal of neuroscience, vol. 15, no. 10, pp. 6651-6665, 1995.

[41] J. Ziburkus, J. Cressman, E. Barreto and S. Schiff, "Interneuron and pyramidal cell interplay during in vitro seizure-like events," Journal of neurophysiology, vol. 95, no. 6, pp. 3948-3954, 2006. 
[42] M. Esclapez, J. Hirsch, R. Khazipov, Y. Ben-Ari and C. Bernard, "Operative GABAergic inhibition in hippocampal CA1 pyramidal neurons in experimental epilepsy," Proceedings of the national academy of sciences of the USA, vol. 94, no. 22, pp. 12151-12156, 1997.

[43] E. Stark, L. Roux, R. Eichler, Y. Senzai, S. Royer and G. Buzsaki, "Pyramidal cell-interneuron interactions underlie hippocampal ripple oscillations," Neuron, vol. 83, no. 2, pp. 467-480, 2014.

[44] N. Dehghani, A. Peyrache, B. Telenczuk, M. Le Van Quyen, E. Halgren, S. Cash, N. Hatsopoulos and A. Destexhe, "Dynamic balance of excitation and inhibition in human and monkey neocortex," Scientific reports, vol. 6, no. 23176, 2016.

[45] J. Engel Jr, "Excitation and inhibition in epilepsy," The Canadian journal of neurological sciences, vol. 23, no. 3, pp. 167-174, 1996.

[46] E. Krook-Magnuson, C. Armstrong, M. Oijala and I. Soltesz, "On-demand optogenetic control of spontaneous seizures in temporal lobe epilepsy," Nature communications, vol. 4, no. 1376, 2012.

[47] A. Chapman, "Glutamate receptors in epilepsy," Progress in brain research, vol. 116, pp. 371383, 1998.

[48] D. Treiman, "GABAergic Mechanisms in Epilepsy," Epilepsia, vol. 42, no. 3, pp. 8-12, 2001.

[49] D. Chong and A. Lerman, "Practice update: Review of anticonvulsant therapy," Current neurology and neuroscience reports, vol. 16, no. 39, 2016.

[50] A. Volterra and J. Meldolesi, "Astrocytes, from brain glue to communication elements: the revolution continues," Nature reviews neuroscience, vol. 6, no. 8, pp. 626-640, 2005.

[51] F. Amzica and M. Steriade, "Neuronal and glial membrane potentials during sleep and paroxysmal oscillations in the neocortex," The journal of neuroscience, vol. 20, no. 17, pp. 6648-6665, 2000.

[52] F. Amzica, M. Massimini and A. Manfridi, "Spatial buffering during slow and paroxysmal cortical networks of glial cells in vivo," The journal of neuroscience, vol. 22, no. 3, pp. 1042-1053, 2002.

[53] H. Kettenmann and B. Ransom, Neuroglia, Oxford: Oxford University Press, 2005.

[54] K. Heuser, K. Szokol and E. Tauboll, "The role of glial cells in epilepsy," Tidsskr Nor Laegeforen, vol. 134, no. 1, pp. 37-41, 2014.

[55] B. Djukic, K. Casper, B. Philpot, L. Chin and K. McCarthy, "Conditional knock-out of Kir4.1 leads to glial membrane depolarization, inhibition of potassium and glutamate uptake, and enhanced short-term synaptic potentiation," Journal of neuroscience, vol. 27, no. 42, pp. 1135411365, 2007.

[56] K. Heuser, T. Eid, F. Lauritzen, A. Thoren, G. Vindedal, E. Taubøll, L. Gjerstad, D. Spencer, O. Ottersen, E. Nagelhus and N. de Lanerolle, "Loss of perivascular Kir4.1 potassium channels in the sclerotic hippocampus of patients with mesial temporal lobe epilepsy," Journal of neuropathology and experimental neurology, vol. 71, no. 9, pp. 814-825, 2012. 
[57] S. Hinterkeuser, W. Schroder, G. Hager, G. Seifert, I. Blumcke, C. Elger, J. Schramm and C. Steinhauser, "Astrocytes in the hippocampus of patients with temporal lobe epilepsy display changes in potassium conductances," European journal of neuroscience, vol. 12, pp. 2087-2096, 2000 .

[58] A. Wallraff, R. Köhling, U. Heinemann, M. Theis, K. Willecke and S. C., "The impact of astrocytic gap junctional coupling on potassium buffering in the hippocampus," Journal of neuroscience, vol. 26, no. 20, pp. 5438-5447, 2006.

[59] J. Wetherington, G. Serrano and R. Dingledine, "Astrocytes in the epileptic brain," Neuron review, vol. 58, pp. 168-178, 2008.

[60] F. Dudek, A. Obenaus and J. Tasker, "Osmolality-induced changes in extracellular volume alter epileptiform bursts independent of chemical synapses in the rat: Importance of non-synaptic mechanisms in hippocampal epileptogenesis," Neuroscience letters, vol. 120, no. 2, pp. 267-270, 1990.

[61] T. Eid, T. Lee, M. Thomas, M. Amiry-Moghaddam, L. Bjørnsen, D. Spencer, P. Agre, O. Ottersen and N. de Lanerolle, "Loss of perivascular aquaporin 4 may underlie deficient water and $\mathrm{K}+$ homeostasis in the human epileptogenic hippocampus," Proceedings of the national academy of sciences of the USA, vol. 102, no. 4, pp. 1193-1198, 2005.

[62] G. Carmignoto and P. Haydon, "Astrocyte calcium signaling and epilepsy," Glia, vol. 60, pp. 1227-1233, 2012.

[63] T. Eid, M. Thomas, D. Spencer, E. Runden-Pran, J. Lai, G. Malthankar, J. Kim, N. Danbolt, O. Ottersen and N. de Lanerolle, "Loss of glutamine synthetase in the human epileptogenic hippocampus possible mechanism for raised extracellular glutamate in mesial temporal lobe epilepsy," Lancet, vol. 363, pp. 28-37, 2004.

[64] G. Perea, M. Navarrete and A. Araque, "Tripartite synapses: astrocytes process and control synaptic information," Cell , vol. 32, no. 8, pp. 421-431, 2009.

[65] T. Fellin, O. Pascual, S. Gobbo, T. Pozzan, P. Haydon and G. Carmignoto, "Neuronal synchrony mediated by astrocytic glutamate through activation of extrasynaptic NMDA receptors," Neuron, vol. 43, no. 5, pp. 729-743, 2004.

[66] N. Allen and B. Barres, "Glia - more than just brain glue," Nature, vol. 457, no. 5, pp. 675-677, 2009.

[67] A. Nimmerjahn, F. Kirchhoff and F. Helmchen, "Resting microglial cells are highly dynamic surveillants of brain parenchyma in vivo," Science, vol. 308, no. 5726, pp. 1314-1318, 2005.

[68] G. Morris, I. Clark, R. Zinn and B. Vissel, "Microglia: a new frontier for synaptic plasticity, learning and memory, and neurodegenerative disease research," Neurobiology of learning and memory, vol. 105, pp. 40-53, 2013.

[69] U. Eyo, M. Murugan and L. Wu, "Microglia-neuron communication in epilepsy," Glia, vol. 65, pp. 5-18, 2017. 
[70] Y. Shigemoto-Mogami, K. Hoshikawa, J. Goldman, Y. Sekino and K. Sato, "Microglia enhance neurogenesis and oligodendrogenesis in the early postnatal subventricular zone," Journal of neuroscience, vol. 34, no. 6, pp. 2231-2243, 2014.

[71] D. Schafer, E. Lehrman and B. Stevens, "The "quad-partite" synapse: Microglia-synapse interactions in the developing and mature CNS," Glia, vol. 61, no. 1, pp. 24-36, 2013.

[72] S. Ben Achour and O. Pascual, "Glia: the many ways to modulate synaptic plasticity," Neurochemistry international, vol. 57, no. 4, pp. 440-445, 2010.

[73] O. Pascual, S. Ben Achour, P. Rostaing, A. Triller and A. Bessis, "Microglia activation triggers astrocyte-mediated modulation of excitatory neurotransmission," Proceedings of the national academy of sciences of the USA, vol. 109, no. 4, pp. 197-205, 2012.

[74] B. Trapp, J. Wujek, G. Criste, W. Jalabi, X. Yin, G. Kidd, S. Stohlman and R. Ransohoff, "Evidence for synaptic stripping by cortical microglia," Glia, vol. 55, no. 4, pp. 360-368, 2007.

[75] H. Wake, A. Moorhouse, S. Jinno, S. Kohsaka and J. Nabekura, "Resting microglia directly monitor the functional state of synapses in vivo and determine the fate of ischemic terminals," The journal of neuroscience, vol. 29, no. 13, pp. 3974-3980, 2009.

[76] D. Schafer, E. Lehrman, A. Kautzman, R. Koyama, A. Mardinly, R. Yamasaki, R. Ransohoff, M. Greenberg, B. Barres and B. Stevens, "Microglia sculpt postnatal neural circuits in an activity and complement-dependent manner," Neuron, vol. 74, no. 4, pp. 691-705, 2012.

[77] K. Biber, H. Neumann, K. Inoue and H. Boddeke, "Neuronal 'On' and 'Off' signals control microglia," Trends in neurosciences, vol. 30, no. 11, pp. 596-602, 2007.

[78] A. Fontainhas, M. Wang, K. Liang, S. Chen, P. Mettu, M. Damani, R. Fariss, W. Li and W. Wong, "Microglial morphology and dynamic behavior is regulated by ionotropic glutamatergic and GABAergic neurotransmission," PLoS One, vol. 6, no. 1, 2011.

[79] I. Ali, D. Chugh and C. Ekdahl, "Role of fractalkine-CX3CR1 pathway in seizure-induced microglial activation, neurodegeneration, and neuroblast production in the adult rat brain," Neurobiology of disease, vol. 74, pp. 194-203, 2015.

[80] C. Lauro, M. Catalano, F. Trettel and C. Limatola, "Fractalkine in the nervous system: neuroprotective or neurotoxic molecule?," Annals of the New York academy of sciences, vol. 1351, pp. 141-148, 2015.

[81] U. Eyo, J. Peng, P. Swiatkowski, A. Mukherjee, A. Bispo and L. Wu, "Neuronal hyperactivity recruits microglial processes via neuronal NMDA receptors and microglial P2Y12 receptors after status epilepticus," Journal of neuroscience, vol. 34, p. 10528-10540, 2014.

[82] N. Derecki, J. Cronk, Z. Lu, E. Xu, S. Abbott, P. Guyenet and J. Kipnis, "Wild-type microglia arrest pathology in a mouse model of Rett syndrome," Nature, vol. 484, no. 7392, pp. 105-109, 2012. 
[83] C. Colton, "Heterogeneity of microglial activation in the innate immune response in the brain," Journal of neuroimmune pharmacology, vol. 4, no. 4, pp. 399-418, 2009.

[84] F. Yang, Z. Liu, J. Chen, S. Zhang, Q. Quan, Y. Huang and W. Jiang, "Roles of astrocytes and microglia in seizure-induced aberrant neurogenesis in the hippocampus of adult rats," Journal of neuroscience research, vol. 88, no. 3, pp. 519-529, 2010.

[85] A. Tort, M. Kramer, C. Thorn, D. Gibson, Y. Kubota, A. Graybiel and N. Kopell, "Dynamic crossfrequency couplings of local field potential oscillations in rat striatum and hippocampus during performance of a T-maze task," Proceedings of the national academy of sciences of the USA, vol. 105, no. 51, pp. 20517-20522, 2008.

[86] J. Lisman and O. Jensen, "The theta-gamma neural code," Neuron, vol. 77, no. 6, pp. 1002-1016, 2013.

[87] J. Lisman, "The theta/gamma discrete phase code occuring during the hippocampal phase precession may be a more general brain coding scheme," Hippocampus, vol. 15, no. 7, pp. 913$922,2005$.

[88] N. Axmacher, M. Henseler, O. Jensen, I. Weinreich, C. Elger and J. Fell, "Cross-frequency coupling supports multi-item working memory in the human hippocampus," Proceedings of the national academy of sciences of the USA, vol. 107, no. 7, pp. 3228-3233, 2009.

[89] F. Mormann, J. Fell, N. Axmacher, B. Weber, K. Lehnertz, C. Elger and G. Fernández, "Phase/amplitude reset and theta-gamma interaction in the human medial temporal lobe during a continuous word recognition memory task," Hippocampus, vol. 15, no. 7, pp. 890-900, 2005.

[90] R. Canolty, E. Edwards, S. Dalal, M. Soltani, S. Nagarajan, H. Kirsch, M. Berger, N. Barbaro and R. Knight, "High gamma power is phase-locked to theta oscillations in human neocortex," Science, vol. 313, no. 5793, pp. 1626-1628, 2006.

[91] A. Tort, R. Komorowski, H. Eichenbaum and N. Kopell, "Measuring phase-amplitude coupling between neuronal oscillations of different frequencies," Journal of neurophysiology, vol. 104, no. 2, pp. 1195-1210, 2010.

[92] S. Colic, R. Wither, M. Lang, L. Zhang, J. Eubanks and B. Bardakjian, "Prediction of antiepileptic drug treatment outcomes using machine learning," Journal of neural engineering, vol. 14, no. 1, 2016.

[93] M. Guirgis, Y. Chinvarun, P. Carlen and B. Bardakjian, "The role of delta-modulated high frequency oscillations in seizure state classification," in Annual conference proceedings of the IEEE engineering in medicine and biology, Osaka, 2013.

[94] S. Colic, M. Lang, R. Wither, L. Zhang, J. Eubanks and B. Bardakjian, "Gene reactivation diminishes delta-modulated high frequency oscillations during seizure-like events in Mecp2deficient mice," in International IEEE/EMBS conference on neural engineering, Montpellier, 2015 . 
[95] T. Hilton, Classification of pre-clinical seizure state transitions in scalp EEG using global crossfrequency coupling index (MASc Thesis), Toronto: University of Toronto, 2017.

[96] K. Edakawa, T. Yanagisawa, H. Kishima, R. Fukuma, S. Oshino, H. Khoo, M. Kobayashi, M. Tanaka and T. Yoshimine, "Detection of epileptic seizures using phase-amplitude coupling in intracranial electroencephalography," Nature scientific reports, vol. 6, no. 25422, 2016.

[97] C. Li, D. Jacobs, T. Hilton, M. del Campo, Y. Chinvarun, P. Carlen and B. Bardakjian, "Epileptogenic source imaging using cross-frequency coupled signals from scalp EEG," IEEE transactions on biomedical engineering, vol. 63, no. 12, pp. 2608-2618, 2016.

[98] F. Wendling, "Computational models of epileptic activity: a bridge between observation and pathophysiological interpretation," Expert review of neurotherapeutics, vol. 8, no. 6, pp. 889-96, 2008.

[99] W. Lytton, "Computer modelling of epilepsy," Nature reviews, vol. 9, no. 8, pp. 626-37, 2008.

[100] F. Wendling, P. Benquet, F. Bartolomei and V. Jirsa, "Computational models of epileptiform activity," Journal of neuroscience methods, vol. 260, pp. 233-251, 2016.

[101] S. Colic, O. Zalay and B. Bardakjian, "Responsive neuromodulators based on artificial neural networks used to control seizure-like events in a computational model of epilepsy," International journal of neural systems, vol. 21, no. 5, p. 367-383, 2011.

[102] O. Zalay and B. Bardakjian, "Synthesis of high-complexity rhythmic signals for closed-loop electrical neuromodulation," Neural networks, vol. 42, pp. 62-73, 2013.

[103] O. Zalay, Cognitive rhythm generators for modelling and modulation of neuronal electrical activity (PhD Thesis), Toronto: University of Toronto, 2011.

[104] V. Marmarelis, "Modeling methodology for nonlinear physiological systems," Annals of biomedical engineering, vol. 25, no. 2, pp. 239-251, 1997.

[105] R. FitzHugh, "Mathematical models of threshold phenomena in the nerve membrane," The bulletin of mathematical biophysics, vol. 17, no. 4, pp. 257-278, 1955.

[106] W. van Drongelen, H. Lee, R. Stevens and M. Hereld, "Propogation of seizure-like activity in a model of neocortex," Journal of clinical neurophysiology, vol. 24, no. 2, pp. 182-188, 2007.

[107] P. Suffczynski, S. Kalitzin and F. Lopes Da Silva, "Dynamics of non-convulsive epileptic phenomena modeled by a bistable neuronal network," Neuroscience, vol. 126, no. 2, pp. 467-484, 2004.

[108] O. Zalay and B. Bardakjian, "Theta phase precession and phase selectivity: a cognitive device description of neural coding," Journal of neural engineering, vol. 6, 2009. 
[109] D. Cosandier-Rimele, F. Bartolomei, I. Merlet, P. Chauvel and F. Wendling, "Recording of fast activity at the onset of partial seizures: depth EEG vs scalp EEG," Neuroimage, vol. 59, pp. 34743487, 2012.

[110] V. Volman, M. Bazhenov and T. Sejnowski, "Computational models of neuron-astrocyte interaction in epilepsy," Frontiers in computational neuroscience, vol. 6, no. 58, 2012.

[111] D. Reato, M. Cammarota, L. Parra and G. Carmignoto, "Computational model of neuron-astrocyte interactions during focal seizure generation," Frontiers in computational neuroscience, vol. 6, no. $81,2012$.

[112] D. Gentiletti, P. Suffczynski, V. Gnatkovsky and M. de Curtis, "Changes of ionic concentrations during seizure transitions - a modelling study," International journal of neural systems, vol. 27, no. $4,2017$.

[113] D. Postnov, L. Ryazanova and O. Sosnovtseva, "Functional modeling of neural-glial interaction," Biosystems, vol. 89, no. 1-3, pp. 84-91, 2007.

[114] B. Bardakjian and N. Diamant, "A mapped clock oscillator model for transmembrane electrical rhythmic activity in excitable cells," Journal of theoretical biology, vol. 166, no. 3, pp. 225-235, 1994.

[115] J. Zariffa, M. Ebden and B. Bardakjian, "A synaptic input portal for a mapped clock oscillator model of neuronal electrical rhythmic activity," Journal of neural engineering, vol. 1, pp. 158-164, 2004.

[116] J. Zariffa and B. Bardakjian, "Neuronal electrical rhythms described by composite mapped clock oscillators," Annals of biomedical engineering, vol. 34, no. 1, pp. 128-141, 2006.

[117] O. Zalay and B. Bardakjian, "Mapped clock oscillators as ring devices and their application to neuronal electrical rhythms," IEEE transactions on neural systems and rehabilitation engineering, vol. 16, no. 3, pp. 233-244, 2008.

[118] G. Mitsis, A. French, U. Höger, S. Courellis and V. Marmarelis, "Principal dynamic mode analysis of action potential firing in a spider mechanoreceptor," Biological cybernetics, vol. 96, no. 1, pp. 113-127, 2007.

[119] O. Zalay, D. Serletis, P. Carlen and B. Bardakjian, "System characterization of neuronal excitability in the hippocampus and its relevance to observed dynamics of spontaneous seizurelike transitions," Journal of neural engineering, vol. 7, 2010.

[120] F. Farah and B. Bardakjian, "High frequency oscillations in a recurrent seizure-like events model," in Society for autonomous neurodynamics, Toronto, 2016.

[121] E. Kang, O. Zalay, D. Serletis, P. Carlen and B. Bardakjian, "Markers of pathological excitability derived from principal dynamic modes of hippocampal neurons," Journal of neural engineering, vol. 9 , no. $5,2012$. 
[122] M. Cotic, A. Chiu, J. S, P. Carlen and B. Bardakjian, "Common time-frequency analysis of local field potential and pyramidal cell activity in seizure-like events of the rat hippocampus," Journal of neural engineering, vol. 8, no. 4, 2011.

[123] C. Moler, "Stiff Differential Equations," MathWorks, 2003. [Online]. Available: https://www.mathworks.com/company/newsletters/articles/stiff-differential-equations.html. [Accessed 29 August 2017].

[124] S. Colic, R. Wither, L. Zhang, J. Eubanks and B. Bardakjian, "Characterization of seizure-like events recorded in vivo in a mouse model of Rett syndrome," Neural networks, vol. 46, pp. 109$115,2013$.

[125] P. Suffczynski, F. Lopes da Silva, J. Parra and S. Kalitzin, "Dynamics of epileptic phenomena determined from statistics of ictal transitions," IEEE transactions on biomedical engineering, vol. 53, no. 3, pp. 524-532, 2006.

[126] G. Vegas-Sánchez-Ferrero, M. Martín-Fernández and J. Miguel Sanches, "A gamma mixture model for IVUS imaging," in Multi-modality atherosclerosis imaging and diagnosis, Springer, 2014, pp. 155-171.

[127] S. Colic, M. Lang, R. Wither, Z. Liang, J. Eubanks and B. Bardakjian, "Characterization of HFOs in short and long duration discharges recorded from in-vivo MeCP2-deficient mice," in Conference proceedings of the IEEE engineering in medicine and biology society, Chicago, 2014.

[128] S. Mylvaganam, M. Ramani, M. Krawczyk and P. Carlen, "Roles of gap junctions, connexins, and pannexins in epilepsy," Frontiers in physiology, vol. 5, no. 172, 2014.

[129] Y. Li, X. Du, C. Liu, Z. Wen and J. Du, "Reciprocal regulation between resting microglial dynamics and neuronal activity in vivo," Developmental cell, vol. 23, no. 6, pp. 1189-1202, 2012.

[130] K. Ji, G. Akgul, L. Wollmuth and S. Tsirka, "Microglia actively regulate the number of functional synapses," PLoS One, vol. 8, no. 2, 2013.

[131] I. Maezawa and L. Jin, "Rett syndrome microglia damage dendrites and synapses by the elevated release of glutamate," Journal of neuroscience, vol. 30, no. 15, pp. 5346-5356, 2010.

[132] E. Dudek, "Epileptogenesis: A new twist on the balance of excitation and inhibition," Epilepsy currents, vol. 9, no. 6, pp. 174-176, 2009.

[133] C. Symonds, "Excitation and inhibition in epilepsy," Proceedings of the royal society of medicine, vol. 52, no. 6, pp. 395-402, 1959.

[134] E. Gamble and R. DiCaprio, "Nonspiking and spiking proprioceptors in the crab: White noise analysis of spiking CB-chordotonal organ afferents," Journal of neurophysiology, vol. 89, no. 4, pp. $1815-1825,2003$.

[135] V. Marmarelis, M. Juusola and A. French, "Principal dynamic mode analysis of nonlinear transduction in a spider mechanoreceptor," Annals of biomedical engineering, vol. 27, no. 3, pp. 391-402, 1999. 
[136] M. Koppert, S. Kalitzin, F. Lopes da Silva and M. Viergever, "Plasticity-modulated seizure dynamics for seizure termination in realistic neuronal models," Journal of neural engineering, vol. 8, no. 4, 2011.

[137] A. Morrison, M. Diesmann and W. Gerstner, "Phenomenological models of synaptic plasticity based on spike timing," Biological cybernetics, vol. 98, no. 6, pp. 459-478, 2008.

[138] P. Mesejo, O. Ibáñez, E. Fernández-Blanco, F. Cedrón, A. Pazos and A. Porto-Pazos, "Artificial neuron-glia networks learning approach based on cooperative coevolution," International journal of neural systems, vol. 25, no. 4, 2015. 


\section{Appendix}

\section{The Microglial Pruning Mechanism}

As described in section 3.1.2 of Chapter 3, the pruning can be described by the following equation:

$$
\rho=1-c_{m: n} P\left(V_{\mu}\right)
$$

where $\rho$ is a value ranging from 0 and 1 that is multiplied by the intra-neuron coupling coefficients, $\mathrm{c}_{\mathrm{m}: \mathrm{n}}$ is the coupling coefficient from microglial CRG $m$ to neuronal $\mathrm{CRG} n$, and $P(V \mu)$ is a sigmoidal pruning function acting on the scaled output of the microglial membrane voltage and taking the following form:

$$
s(u)=\frac{1}{1+\exp \left(-c_{1}\left(u-c_{2}\right)\right)}
$$

where $u$ is the input to the sigmoid, and $c_{1}$ and $c_{2}$ are function-dependent constants. As such, the effect of the pruning can be tuned in two ways: the sigmoid and the microglia-neuron coupling coefficient.

In the sigmoid, the steepness constant, $\mathrm{c}_{1}$, has been set to 1 such that it scales to the microglial voltage output (Fig. A.1). The threshold constant, $\mathrm{c}_{2}$, can be varied to control how easily the

pruning is triggered. As can be seen in Fig. A.1b, if $c_{2}$ is small, then the microglial voltage will usually be larger and the output of the sigmoid will thus be close to 1 . In this case, it will be easy to trigger the pruning effect. If $c_{2}$ is high, the microglial voltage output may not reach it, and the sigmoid output will be closer to 0 . In this case, it will be more difficult to trigger the pruning effect. This formulation emulates the activity-dependent nature of microglial synapse stripping. Physiologically, the microglia survey the surrounding environment and prune the synapses based on their activity level. In the model, the microglia receive input from the neurons and this then influences the amplitude of the voltage output. This voltage output is then compared with the threshold constant of the sigmoid and the level of pruning is correspondingly decided. 
The microglia-neuron coupling coefficient is used to control how strong or effective the pruning effect will be on the neurons. The larger the coupling coefficient, the stronger the connection between the microglia and the neurons, and the more effective the pruning will be. Following seizures, there was evidence of a) increased numbers of microglia, and b) increased fractalkine signaling between the microglia and the neurons [69]. This suggests stronger association between microglia and neurons in epilepsy, and warrants a high coupling value in the network model under hyperexcitable conditions.

To summarize, maximal pruning will occur when both the sigmoidal output and the coupling coefficient are equal to 1 . In that case, $\rho=0$, and the connections between the pyramidal cells and interneurons will be entirely severed and the synapses entirely pruned. 


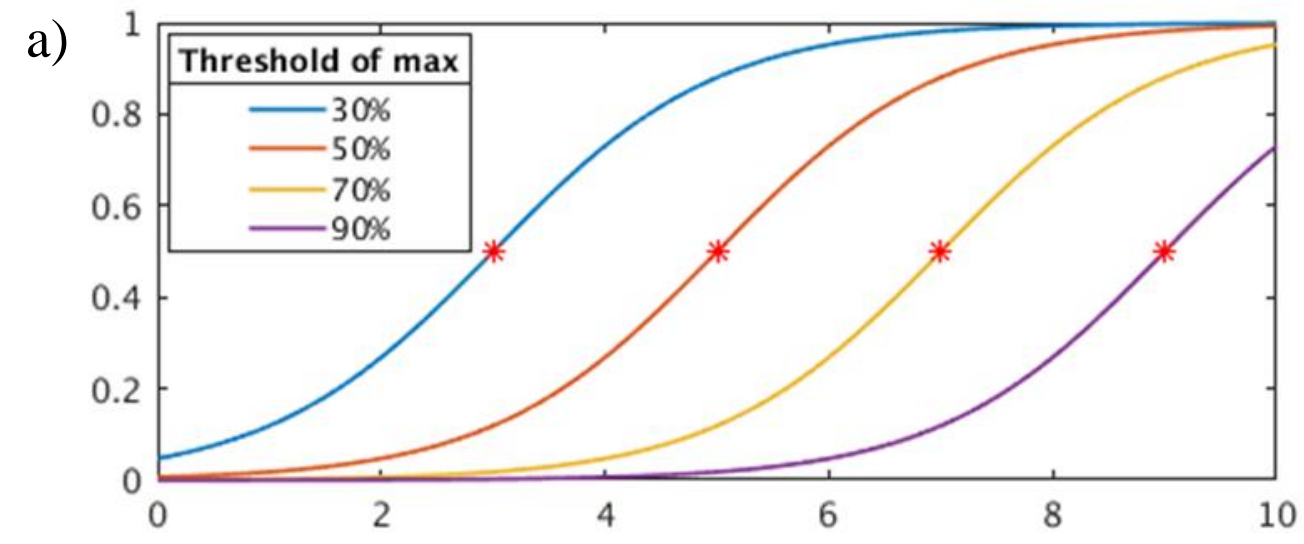

b)

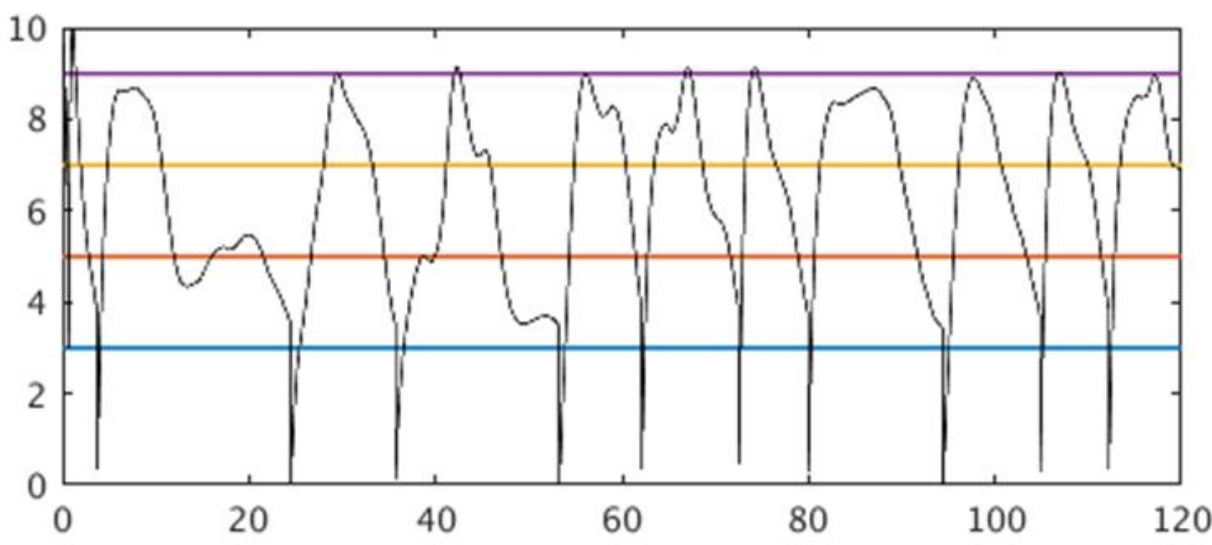

Fig. A.1. The sigmoidal pruning mechanism. a) The family of sigmoidal functions as the threshold constant $\mathrm{c}_{2}$ is varied from 3 to 9 (30\% to $90 \%$ ). b) The thresholds corresponding to the sigmoidal functions in a) are superimposed on the scaled output of the microglial voltage. The larger the threshold, the less often the microglial voltage can reach it, and thus the more difficult it is to trigger the pruning mechanism. 